\title{
EXISTENCE OF COMPLEX STRUCTURES ON DECOMPOSABLE LIE ALGEBRAS
}

\author{
BY MARCIN SROKA
}

\begin{abstract}
We provide the classification of the six-dimensional decomposable Lie algebras, with the dimension of the biggest indecomposable summand less than five, admitting complex structures.
\end{abstract}

1. Introduction. An almost complex structure on a Lie algebra $\mathfrak{h}$ is an endomorphism

$$
\mathcal{I}: \mathfrak{h} \rightarrow \mathfrak{h}
$$

such that

$$
\mathcal{I}^{2}=-i d
$$

We say that an almost complex structure $\mathcal{I}$ on $\mathfrak{h}$ is a complex structure if the following condition, being a linear version of the vanishing of the Nijenhuis tensor, holds

$$
[\mathcal{I}, \mathcal{I}](v, w)=-[v, w]+[\mathcal{I} v, \mathcal{I} w]-\mathcal{I}[\mathcal{I} v, w]-\mathcal{I}[v, \mathcal{I} w]=0
$$

f or any $v, w \in \mathfrak{h}$. Because of the trivialization of the tangent bundle, by left invariant vector fields, (almost) complex structures on a Lie algebra correspond to left invariant (almost) complex structures on any associated Lie group or its quotient by a discrete subgroup.

The following approach is classic. To any $\mathcal{I}$ as above we can assign its extension $\mathcal{I}^{\mathbb{C}}$ to $\mathfrak{h}^{\mathbb{C}}$, being a complexification of $\mathfrak{h}$, and vector subspaces

$$
\begin{gathered}
\mathfrak{h}_{\mathcal{I}}^{1,0}=\left\{u \in \mathfrak{h}^{\mathbb{C}} \mid \mathcal{I}^{\mathbb{C}}(u)=i u\right\}=\{h-i \mathcal{I}(h) \mid h \in \mathfrak{h}\} \\
\mathfrak{h}_{\mathcal{I}}^{0,1}=\left\{u \in \mathfrak{h}^{\mathbb{C}} \mid \mathcal{I}^{\mathbb{C}}(u)=-i u\right\}=\{h+i \mathcal{I}(h) \mid h \in \mathfrak{h}\}
\end{gathered}
$$

2010 Mathematics Subject Classification. 17B40, 53C15, 53C30.

Key words and phrases. Invariant complex structures, Lie algebras, homogeneous manifolds. 
of $\mathfrak{h}^{\mathbb{C}}$ such that

$$
\mathfrak{h}^{\mathbb{C}}=\mathfrak{h}_{\mathcal{I}}^{1,0} \oplus \mathfrak{h}_{\mathcal{I}}^{0,1} \quad \text { and } \quad \mathfrak{h}_{\mathcal{I}}^{1,0}=\overline{\mathfrak{h}_{\mathcal{I}}^{0,1}}
$$

Actually, almost complex structures correspond bijectively to complex subspaces $\mathfrak{q}$ of $\mathfrak{h}^{\mathbb{C}}$ such that

$$
\mathfrak{h}^{\mathbb{C}}=\mathfrak{q} \oplus \overline{\mathfrak{q}} .
$$

The following definitions and properties are standard.

Definition 1.1. Let $\mathcal{I}$ be an almost complex structure on $\mathfrak{h}$.

- $\mathcal{I}$ is a complex structure if

$$
-[v, w]+[\mathcal{I} v, \mathcal{I} w]-\mathcal{I}[\mathcal{I} v, w]-\mathcal{I}[v, \mathcal{I} w]=0 \text { for any } v, w \in \mathfrak{h}
$$

which is equivalent to $\mathfrak{h}_{\mathcal{I}}^{1,0}$ being a subalgebra of $\mathfrak{h}^{\mathbb{C}}$, i.e., $\left[\mathfrak{h}_{\mathcal{I}}^{1,0}, \mathfrak{h}_{\mathcal{I}}^{1,0}\right] \subset \mathfrak{h}_{\mathcal{I}}^{1,0}$.

- $\mathcal{I}$ is an abelian complex structure if

$$
[\mathcal{I} v, \mathcal{I} w]=[v, w] \text { for any } v, w \in \mathfrak{h}
$$

which is equivalent to $\mathfrak{h}_{\mathcal{I}}^{1,0}$ being an abelian subalgebra.

- $\mathcal{I}$ is a bi-invariant complex structure if

$$
\mathcal{I}[v, w]=[\mathcal{I} v, w] \text { for any } v, w \in \mathfrak{h}
$$

which is equivalent to $\mathfrak{h}_{\mathcal{I}}^{1,0}$ being an ideal, i.e., $\left[\mathfrak{h}_{\mathcal{I}}^{1,0}, \mathfrak{h}^{\mathbb{C}}\right] \subset \mathfrak{h}_{\mathcal{I}}^{1,0}$.

Let us just note that only the last type of a complex structure, namely the bi-invariant one, turns a corresponding Lie group into a complex Lie group since in the other cases we do not know whether right multiplications are holomorphic mappings with respect to $\mathcal{I}$ or not.

Characterization of Lie algebras admitting a complex structure is an established problem. Probably the first general result in this direction was Samelson's [20] (see also [24] for results on compact complex homogeneous spaces with transitive action of a compact Lie group) who proved that every compact Lie group admits an invariant complex structure. It was proven by Morimoto [13] that any reductive, i.e. being a direct sum of an abelian and semi-simple ones, Lie algebra does admit a complex structure. Geometry of invariant complex structures on reductive Lie groups was then studied by Snow in $[22]$.

Since the precise, algebraic, description of Lie algebras admitting complex structures seems to be beyond the grasp, the interest was recently directed into classification results in low dimensions. In dimension four the classification is known for solvable algebras. It follows from the Snow's paper [23] for solvable algebras with the commutator of dimension less than three and Ovando's [17] for those with a three dimensional commutator. Since there are only few nonsolvable four dimensional Lie algebras, the classification is actually complete for dimension four. The method of both these papers relies on an equivalent 
description of a complex structure in terms of a complex subalgebra as in Definition 1.1. In this case such an attempt is very fruitful since there are only two dimensional Lie algebras and consequently only two candidates for these complex subalgebras. Even in the next possible dimension, which is six, the difficulty grows up rapidly because there are infinitely many three dimensional Lie algebras of at least eight significantly different types, cf. Theorem 2.4.

The classification in dimension six is known only for nilpotent algebras due to a Salamon's influential paper [19]. There are 34 non-isomorphic algebras of the type and only 18 of them admit complex structures, and product algebras, i.e. of the form $\mathfrak{g} \times \mathfrak{g}$, due to the article [9]. Salamon's methods are designed purely for nilpotent algebras and rely on analysis performed in special bases. Recently his work has been completed by Ceballos, Otal, Ugarte and Villacampa in $[\mathbf{7}]$, where they provided the description of moduli spaces of complex structures for those nilpotent algebras.

Because of the difficulties described above the attention has recently been directed to classifying the Lie algebras admitting special types of complex structures. For the abelian complex structures on nilpotent Lie algebras the classification is known up to dimension six, due to Andrada, Barberis and Dotti's paper $[\mathbf{1}]$. In $[\mathbf{1 0}]$ the classification of six-dimensional solvable Lie algebras admitting complex structures such that the associated solvmanifold has a trivial canonical bundle was obtained. The classification of solvable sixdimensional algebras admitting the complex structures of splitting type was conducted in $[\mathbf{3}]$. The problem, conceptually related to our considerations, of classifying the decomposable six-dimensional Lie algebras admitting the halfflat structures was discussed in $[\mathbf{1 1}, \mathbf{2 1}]$.

Although the classification problem is interesting by itself, it gains additional attention because of immediate applications in complex geometry. More concretely, homogeneous manifolds, and, more generally, nil- or solvmanifolds, form an important class of examples, counterexamples or simply toy models on which precise calculations can be carried out in contrast with some abstract manifold. For the concrete examples of some solvmanifolds associated with the solvable algebras covered by our classification, the reader is referred to [6]. Topological or even geometric data about these spaces can be obtained, up to some level, from a pure linear algebra of an associated Lie algebra.

In this paper we intend to broaden the classification in dimension six. The main observation we use is that the existence of a complex structure on a decomposable Lie algebra implies the existence of what is known as the zero torsion endomorphism on each component of the decomposition. This is an observation by Magnin, cf. [12], who used it to describe the space of complex structures on $S U(2) \times S U(2)$. By contrast, we plan to use the existence of zero 
torsion endomorphism as an obstruction to the existence of a complex structure. This precludes many decomposable algebras from admitting complex structures but plays only the role of an obstacle, since it sometimes happens that all the components do admit a zero torsion endomorphism yet the algebra under consideration still does not admit a complex structure. In such a case closer analysis of possible forms of a zero torsion endomorphism must be provided, see for example the proof of Proposition 4.6. The method also gives us an additional gain since once we prove the algebra $\mathfrak{g}$ does not admit a zero torsion endomorphism we know that $\mathfrak{g} \times \mathfrak{h}$ does not admit a complex structure for any Lie algebra $\mathfrak{h}$. This by hand provides results on high dimensional algebras which is in contrast with the usually obtained results. On the other hand, proving that a given algebra does not admit a zero torsion endomorphism is quite involved computationally because it requires showing that a given system of quite some polynomial, non-homogeneous (in general), equations does not have a solution.

In Section 2 we carry the classification for algebras of the form $\mathfrak{g}_{3} \times \mathfrak{h}_{3}$ where $\mathfrak{g}_{3}$ and $\mathfrak{h}_{3}$ are three dimensional Lie algebras. In a special cases when $\mathfrak{g}_{3} \cong \mathfrak{h}_{3}$ this was achieved in [9], proofs of some results in that case are simplified in comparison with that reference. Section 3 contains a review of the classification in dimension four, since this will be used in Section 4 where we deal with the case when the biggest indecomposable summand of the decomposition is of dimension four. This is the main part of the paper. When the dimension of the indecomposable summand is 5 , the resulting systems of equations seems to be too complicated so our method is useless in that case, i.e. in general we are not able to show the non-existence of a zero torsion endomorphism on a five dimensional indecomposable algebra.

Acknowledgments: During the preparation of the paper the author was partially supported by Polish National Science Centre Grant No. 2020/36/T/ ST1/00334.

2. Complex structures on Lie algebras of the form $\mathfrak{g}_{3} \times \mathfrak{h}_{3}$. We start by recalling a simple lemma which relates the existence of a complex structure on a decomposable Lie algebra with a system of homomorphisms of the summands. Its verification is an easy computation.

Lemma 2.1 (Lemma 3. in [12]). Suppose $\mathcal{I}$ is a complex structure on a decomposable Lie algebra

$$
\mathfrak{g}=\mathfrak{g}_{1} \times \ldots \times \mathfrak{g}_{n} .
$$

Let $p_{i}$ be a projection of $\mathfrak{g}$ onto $\mathfrak{g}_{i}$ and let $q_{i}$ be an inclusion of $\mathfrak{g}_{i}$ into $\mathfrak{g}$, then for

$$
\mathcal{I}_{i}^{j}=p_{j} \circ \mathcal{I} \circ q_{i}: \mathfrak{g}_{i} \rightarrow \mathfrak{g}_{j}
$$


there is

$$
\left[\mathcal{I}_{i}^{i}, \mathcal{I}_{i}^{i}\right](v, w)=-[v, w]+\left[\mathcal{I}_{i}^{i} v, \mathcal{I}_{i}^{i} w\right]-\mathcal{I}_{i}^{i}\left[\mathcal{I}_{i}^{i} v, w\right]-\mathcal{I}_{i}^{i}\left[v, \mathcal{I}_{i}^{i} w\right]=0
$$

and

$$
\mathcal{I}_{i}^{j}\left[\mathcal{I}_{i}^{i} v, w\right]+\mathcal{I}_{i}^{j}\left[v, \mathcal{I}_{i}^{i} w\right]=\left[\mathcal{I}_{i}^{j} v, \mathcal{I}_{i}^{j} w\right]
$$

for any $v, w \in \mathfrak{g}_{i}$ and $i \neq j$.

Definition $2.2([\mathbf{1 2}])$. A zero torsion endomorphism $\mathcal{I}$ on a Lie algebra $\mathfrak{h}$ is one with the property

$$
[\mathcal{I}, \mathcal{I}](v, w)=0
$$

for all $v, w \in \mathfrak{h}$.

REMARK 2.3. By Lemma 2.1 a necessary condition for the existence of a complex structure on a Lie algebra

$$
\mathfrak{g}=\mathfrak{g}_{1} \times \ldots \times \mathfrak{g}_{n}
$$

is that each $\mathfrak{g}_{i}$ carries a zero torsion endomorphism. Notice that a zero endomorphism is never a zero torsion endomorphism unless the algebra under consideration is abelian. It will be seen later that existence of a zero torsion endomorphism is a nontrivial property and is a substantial obstacle to the existence of complex structures.

We would now like to start the classification in the case of algebras of the form $\mathfrak{g}_{3} \times \mathfrak{h}_{3}$. For that purpose let us remind the well known classification of three dimensional Lie algebras proposed by Bianchi. A contemporary source of the classification of Lie algebras up to dimension five is for example [18], although this is not the original reference for any of these dimensions.

THEOREM 2.4 (Bianchi [4]). Every three dimensional real Lie algebra $\mathfrak{g}=$ $\operatorname{span}\left\{e_{1}, e_{2}, e_{3}\right\}$ is isomorphic to exactly one of the following

$$
\begin{aligned}
& A_{3,1}:\left[e_{1}, e_{3}\right]=0,\left[e_{2}, e_{3}\right]=0,\left[e_{1}, e_{2}\right]=0 \\
& A_{3,2}:\left[e_{1}, e_{3}\right]=0,\left[e_{2}, e_{3}\right]=0,\left[e_{1}, e_{2}\right]=e_{1} \\
& A_{3,3}:\left[e_{1}, e_{3}\right]=0,\left[e_{2}, e_{3}\right]=0,\left[e_{1}, e_{2}\right]=e_{3} \\
& A_{3,4}:\left[e_{1}, e_{3}\right]=e_{1},\left[e_{2}, e_{3}\right]=e_{2},\left[e_{1}, e_{2}\right]=0 \\
& A_{3,5}^{\theta}:\left[e_{1}, e_{3}\right]=e_{1},\left[e_{2}, e_{3}\right]=\theta e_{2},\left[e_{1}, e_{2}\right]=0 \text { for }-1 \leq \theta<1, \theta \neq 0 \\
& A_{3,6}:\left[e_{1}, e_{3}\right]=e_{1},\left[e_{2}, e_{3}\right]=e_{1}+e_{2},\left[e_{1}, e_{2}\right]=0 \\
& A_{3,7}^{\theta}:\left[e_{1}, e_{3}\right]=\theta e_{1}-e_{2},\left[e_{2}, e_{3}\right]=e_{1}+\theta e_{2},\left[e_{1}, e_{2}\right]=0 \text { for } \theta \geq 0 \\
& A_{3,8}:\left[e_{1}, e_{3}\right]=e_{2},\left[e_{2}, e_{3}\right]=-e_{1},\left[e_{1}, e_{2}\right]=e_{3} \\
& A_{3,9}:\left[e_{1}, e_{3}\right]=-e_{2},\left[e_{2}, e_{3}\right]=e_{1},\left[e_{1}, e_{2}\right]=e_{3}
\end{aligned}
$$


The next two theorems preclude some of these algebras from admitting a zero torsion endomorphism.

Proposition 2.5. There is no zero torsion endomorphism on the Lie algebra $A_{3,6}$.

Proof. We argue by contradiction. Suppose that there is a zero torsion endomorphism $\mathcal{I}$ on $A_{3,6}$ given in the basis from Theorem 2.4 by the matrix

$$
\left[\begin{array}{ccc}
a & \alpha & k \\
b & \beta & l \\
c & \gamma & m
\end{array}\right] .
$$

Let us write down the torsion conditions. In what follows we use the convention

$$
\begin{aligned}
& \sum a e_{i}=a e_{1}+b e_{2}+c e_{3} \text { etc. } \\
{[\mathcal{I}, \mathcal{I}]\left(e_{1}, e_{2}\right)=} & -\left[e_{1}, e_{2}\right]+\left[\sum a e_{i}, \sum \alpha e_{i}\right]-\mathcal{I}\left[\sum a e_{i}, e_{2}\right]-\mathcal{I}\left[e_{1}, \sum \alpha e_{i}\right] \\
= & a \gamma e_{1}+b \gamma e_{1}+b \gamma e_{2}-c \alpha e_{1}-c \beta e_{1}-c \beta e_{2} \\
& -\mathcal{I}\left(-c e_{1}-c e_{2}\right)-\mathcal{I}\left(\gamma e_{1}\right) \\
= & a \gamma e_{1}+b \gamma e_{1}+b \gamma e_{2}-c \alpha e_{1}-c \beta e_{1}-c \beta e_{2} \\
& +c\left(\sum a e_{i}+\sum \alpha e_{i}\right)-\gamma\left(\sum a e_{i}\right) \\
= & (a \gamma+b \gamma-c \alpha-c \beta+c a+c \alpha-\gamma a) e_{1} \\
& +(b \gamma-c \beta+c b+c \beta-\gamma b) e_{2}+\left(c^{2}+c \gamma-\gamma c\right) e_{3} \\
{[\mathcal{I}, \mathcal{I}]\left(e_{1}, e_{3}\right)=} & -\left[e_{1}, e_{3}\right]+\left[\sum a e_{i}, \sum k e_{i}\right]-\mathcal{I}\left[\sum a e_{i}, e_{3}\right]-\mathcal{I}\left[e_{1}, \sum k e_{i}\right] \\
= & -e_{1}+a m e_{1}+b m e_{1}+b m e_{2}-c k e_{1}-c l e_{1}-c l e_{2} \\
& -\mathcal{I}\left(a e_{1}+b e_{1}+b e_{2}\right)-\mathcal{I}\left(m e_{1}\right) \\
= & -e_{1}+a m e_{1}+b m e_{1}+b m e_{2}-c k e_{1}-c l e_{1}-c l e_{2} \\
& -(a+b)\left(\sum a e_{i}\right)-b\left(\sum \alpha e_{i}\right)-m\left(\sum a e_{i}\right) \\
= & \left(-1+a m+b m-c k-c l-a^{2}-a b-b \alpha-m a\right) e_{1} \\
& +\left(b m-c l-a b-b^{2}-b \beta-m b\right) e_{2}-(a c+b c+b \gamma+m c) e_{3} \\
{[\mathcal{I}, \mathcal{I}]\left(e_{2}, e_{3}\right)=} & -\left[e_{2}, e_{3}\right]+\left[\sum \alpha e_{i}, \sum k e_{i}\right]-\mathcal{I}\left[\sum \alpha e_{i}, e_{3}\right]-\mathcal{I}\left[e_{2}, \sum k e_{i}\right] \\
= & -e_{1}-e_{2}+\alpha m e_{1}+\beta m e_{1}+\beta m e_{2}-\gamma k e_{1}-\gamma l e_{1}-\gamma l e_{2} \\
& -\mathcal{I}\left(\alpha e_{1}+\beta e_{1}+\beta e_{2}\right)-\mathcal{I}\left(m e_{1}+m e_{2}\right) \\
&
\end{aligned}
$$




$$
\begin{aligned}
= & -e_{1}-e_{2}+\alpha m e_{1}+\beta m e_{1}+\beta m e_{2}-\gamma k e_{1}-\gamma l e_{1}-\gamma l e_{2} \\
& -(\alpha+\beta)\left(\sum a e_{i}\right)-\beta\left(\sum \alpha e_{i}\right)-m\left(\sum a e_{i}+\sum \alpha e_{i}\right) \\
= & (-1+\alpha m+\beta m-\gamma k-\gamma l-\alpha a-\beta a-\beta \alpha-m a-m \alpha) e_{1} \\
& +\left(-1+\beta m-\gamma l-\alpha b-\beta b-\beta^{2}-m b-m \beta\right) e_{2} \\
& -(\alpha c+\beta c+\beta \gamma+m c+m \gamma) e_{3}
\end{aligned}
$$

After some obvious manipulations this leads to the following system of equations

$$
\left\{\begin{array}{l}
c=0 \\
b \gamma=0 \\
a b+b^{2}+b \beta=0 \\
1-b m+a^{2}+a b+b \alpha=0 \\
\gamma(\beta+m)=0 \\
1+\gamma l+\alpha b+\beta b+\beta^{2}+m b=0 \\
1-\beta m+\gamma k+\gamma l+a \alpha+a \beta+\beta \alpha+m a=0
\end{array}\right.
$$

From the fourth equation we see that $b \neq 0$, this implies that $\gamma=0$ and $a+b+\beta=0$ from the second and the third equations respectively. Substituting $a=-(b+\beta)$ in the fourth and the last but one equations gives us

$$
\left\{\begin{array}{l}
1-b m+b \beta+\beta^{2}+b \alpha=0 \\
1+\alpha b+\beta b+\beta^{2}+m b=0
\end{array} .\right.
$$

Subtracting this two equations results in $b m=0$, consequently $m=0$. Taking all of this into account allows us to write the last two equations of the first system as

$$
\left\{\begin{array}{l}
1+\alpha b+\beta b+\beta^{2}=0 \\
1-\alpha b-b \beta-\beta^{2}=0
\end{array}\right.
$$

which is a contradiction.

Proposition 2.6. There are no zero torsion endomorphisms on Lie algebras $A_{3,5}^{\theta}$ for $\theta \neq-1$.

Proof. Again we argue by contradiction. Suppose that there is a zero torsion endomorphism $\mathcal{I}$ on $A_{3,5}^{\theta}$ given in the basis from Theorem 2.4 by the matrix

$$
\left[\begin{array}{ccc}
a & \alpha & k \\
b & \beta & l \\
c & \gamma & m
\end{array}\right]
$$

The torsion conditions are 


$$
\begin{aligned}
& {[\mathcal{I}, \mathcal{I}]\left(e_{1}, e_{2}\right)=-\left[e_{1}, e_{2}\right]+\left[\sum a e_{i}, \sum \alpha e_{i}\right]-\mathcal{I}\left[\sum a e_{i}, e_{2}\right]-\mathcal{I}\left[e_{1}, \sum \alpha e_{i}\right]} \\
& =a \gamma e_{1}+b \theta \gamma e_{2}-c \alpha e_{1}-c \theta \beta e_{2}-\mathcal{I}\left(-\theta c e_{2}\right)-\mathcal{I}\left(\gamma e_{1}\right) \\
& =a \gamma e_{1}+b \theta \gamma e_{2}-c \alpha e_{1}-c \theta \beta e_{2}+c \theta\left(\sum \alpha e_{i}\right)-\gamma\left(\sum a e_{i}\right) \\
& =(a \gamma-c \alpha+c \theta \alpha-\gamma a) e_{1}+(b \theta \gamma-c \theta \beta+c \theta \beta-\gamma b) e_{2} \\
& +(c \theta \gamma-\gamma c) e_{3} \\
& {[\mathcal{I}, \mathcal{I}]\left(e_{1}, e_{3}\right)=-\left[e_{1}, e_{3}\right]+\left[\sum a e_{i}, \sum k e_{i}\right]-\mathcal{I}\left[\sum a e_{i}, e_{3}\right]-\mathcal{I}\left[e_{1}, \sum k e_{i}\right]} \\
& =-e_{1}+a m e_{1}+b \theta m e_{2}-c k e_{1}-c \theta l e_{2}-\mathcal{I}\left(a e_{1}+\theta b e_{2}\right)-\mathcal{I}\left(m e_{1}\right) \\
& =-e_{1}+a m e_{1}+b \theta m e_{2}-c k e_{1}-c \theta l e_{2} \\
& -a\left(\sum a e_{i}\right)-b \theta\left(\sum \alpha e_{i}\right)-m\left(\sum a e_{i}\right) \\
& =\left(-1+a m-c k-a^{2}-b \theta \alpha-m a\right) e_{1} \\
& +(b \theta m-c \theta l-a b-\theta b \beta-m b) e_{2}-(a c+b \theta \gamma+m c) e_{3} \\
& {[\mathcal{I}, \mathcal{I}]\left(e_{2}, e_{3}\right)=-\left[e_{2}, e_{3}\right]+\left[\sum \alpha e_{i}, \sum k e_{i}\right]-\mathcal{I}\left[\sum \alpha e_{i}, e_{3}\right]-\mathcal{I}\left[e_{2}, \sum k e_{i}\right]} \\
& =-\theta e_{2}+\alpha m e_{1}+\beta \theta m e_{2}-\gamma k e_{1}-\gamma \theta l e_{2} \\
& -\mathcal{I}\left(\alpha e_{1}+\beta \theta e_{2}\right)-\mathcal{I}\left(\theta m e_{2}\right) \\
& =-\theta e_{2}+\alpha m e_{1}+\beta \theta m e_{2}-\gamma k e_{1}-\gamma \theta l e_{2} \\
& -\alpha\left(\sum a e_{i}\right)-\beta \theta\left(\sum \alpha e_{i}\right)-m \theta\left(\sum \alpha e_{i}\right) \\
& =(\alpha m-\gamma k-\alpha a-\beta \theta \alpha-m \theta \alpha) e_{1} \\
& +\left(-\theta+\beta \theta m-\gamma \theta l-\alpha b-\beta^{2} \theta-m \theta \beta\right) e_{2} \\
& -(\alpha c+\beta \theta \gamma+m \theta \gamma) e_{3} \text {. }
\end{aligned}
$$

We write these conditions as the following system

$$
\left\{\begin{array}{l}
c \alpha=b \gamma=c \gamma=0 \\
1+c k+a^{2}+b \theta \alpha=0 \\
b \theta m-c \theta l-a b-\theta b \beta-m b=0 \\
a c+b \theta \gamma+m c=0 \\
\alpha m-\gamma k-\alpha a-\beta \theta \alpha-m \theta \alpha=0 \\
\theta+\gamma \theta l+\alpha b+\beta^{2} \theta=0 \\
\alpha c+\beta \theta \gamma+m \theta \gamma=0
\end{array}\right.
$$


Suppose that $\alpha=0$. From the second equation we see that $k \neq 0$ and consequently from the fifth equation we conclude $\gamma=0$. This leads to the last but one equation stating $\theta+\theta \beta^{2}=0$; an obvious absurd so we must have $\alpha \neq 0$. From this and the first equation we obtain $c=0$. Again using the second equation we see that $b \neq 0$ and finally, from the first equation, $\gamma=0$. The remaining nontrivial equations are displayed below.

$$
\left\{\begin{array}{l}
1+a^{2}+b \theta \alpha=0 \\
\theta m-a-\theta \beta-m=0 \\
m-a-\beta \theta-m \theta=0 \\
\theta+\alpha b+\beta^{2} \theta=0
\end{array}\right.
$$

Adding the second and the third equations gives $a=-\beta \theta$; applying this in the first equation and multiplying the last one by $\theta$ results in

$$
\left\{\begin{array}{l}
1+\beta^{2} \theta^{2}+b \theta \alpha=0 \\
\theta^{2}+\alpha b \theta+\beta^{2} \theta^{2}=0
\end{array}\right.
$$

which of course means that $\theta^{2}=1$; a contradiction.

Remark 2.7. It follows from Propositions 2.5, 2.6 and Remark 2.3 that there are no complex structures on $A_{3,5}^{\theta} \times \mathfrak{h}$ and $A_{3,6} \times \mathfrak{h}$ for $0<|\theta|<1$ and any Lie algebra $\mathfrak{h}$.

As the next theorem shows, the Lie algebra $A_{3,5}^{-1}$ is of a type different from the Lie algebras from the last remark, since it does admit a zero torsion endomorphism in contrast with $A_{3,5}^{\theta}$ for $\theta \neq-1$ or $A_{3,6}$, and the other algebras from the Theorem 2.4, since, as it will be stated in Remark 2.13, all of those admit a normal almost contact structure, in contrast to $A_{3,5}^{-1}$.

Proposition 2.8. For a three dimensional real Lie algebra $\mathfrak{h}$, the Lie algebra $A_{3,5}^{-1} \times \mathfrak{h}$ admits a complex structure if and only if $\mathfrak{h}$ is isomorphic to $A_{3,7}^{0}$.

Proof. Firstly we define a complex structure on $A_{3,5}^{-1} \times A_{3,7}^{0}$. Let $e_{1}, e_{2}$, $e_{3}$ and $f_{1}, f_{2}, f_{3}$ be bases for $A_{3,5}^{-1}$ and $A_{3,7}^{0}$ respectively as in Theorem 2.4. Consider a complex structure $\mathcal{J}$ given by

$$
\begin{aligned}
& e_{1} \longmapsto e_{2}+f_{1}, \\
& e_{2} \longmapsto e_{1}+f_{2}, \\
& e_{3} \longmapsto f_{3} .
\end{aligned}
$$

We should check that

$$
\left\{g-i \mathcal{J} g: g \in A_{3,5}^{-1} \times A_{3,7}^{0}\right\} \subset\left(A_{3,5}^{-1} \times A_{3,7}^{0}\right)^{\mathbb{C}}
$$


is a complex subalgebra. Since

$$
\left\{g-i \mathcal{J} g: g \in A_{3,5}^{-1} \times A_{3,7}^{0}\right\}=\operatorname{span}\left\{u_{1}, u_{2}, u_{3}\right\}
$$

where

$$
\begin{aligned}
& u_{1}=e_{1}-i e_{2}-i f_{1}, \\
& u_{2}=e_{2}-i e_{1}-i f_{2}, \\
& u_{3}=e_{3}-i f_{3}
\end{aligned}
$$

it is enough to observe that

$$
\begin{gathered}
{\left[u_{1}, u_{2}\right]=0} \\
{\left[u_{1}, u_{3}\right]=\left[e_{1}-i e_{2}-i f_{1}, e_{3}-i f_{3}\right]=i\left(e_{2}-i e_{1}-i f_{2}\right)=i u_{2},} \\
{\left[u_{2}, u_{3}\right]=\left[e_{2}-i e_{1}-i f_{2}, e_{3}-i f_{3}\right]=-i\left(e_{1}-i e_{2}-i f_{1}\right)=-i u_{1} .}
\end{gathered}
$$

For the proof of the second implication we argue as in [9]. Since the reasoning is exactly the same as in the part "Proof of the non-existence of integrable complex structures - case (4) with $\theta \neq 1$ " of that paper, we only write down the main steps and the conclusion. Suppose there is a complex structure $\mathcal{I}$ on $A_{3,5}^{-1} \times \mathfrak{h}$. One can prove the existence of a basis $u, v, w$ of $A_{3,5}^{-1}$ such that $u^{\prime}=\mathcal{I}_{1}^{2} u, v^{\prime}=\mathcal{I}_{1}^{2} v, w^{\prime}=\mathcal{I}_{1}^{2} w$ is a basis of $\mathfrak{h}$. What is more, $\left[v^{\prime}, w^{\prime}\right]=0$ and the adjoint endomorphism $\left[\cdot, u^{\prime}\right]: \mathfrak{h} \rightarrow \mathfrak{h}$ has eigenvalues $i$ and $-i$. From the Theorem 2.4, it is easy to see that the only algebra satisfying this requirements is $A_{7}^{0}$.

It turns out that the products of any of the algebras not covered so far do admit complex structures. What is more, any of these products admit a complex structures canonically associated to an almost contact structure. Reader unfamiliar with almost contact structures may consult a survey on them that can be found in [5]. Again, we will be focused on the linear counterpart of the theory of invariant structures of this kind on homogeneous manifolds. Let us just state the definition for clarity.

Definition 2.9. An almost contact structure $\left(\mathfrak{h}, \phi, u^{*}, v\right)$ on a Lie algebra $\mathfrak{h}$ is an endomorphism $\phi$, a co-vector $u^{*}$ and a vector $v$ such that

$$
\phi^{2}=-i d+v u^{*} \text { and } u^{*}(v)=1 .
$$

The structure $\left(\mathfrak{h}, \phi, u^{*}, v\right)$ is normal if

$$
N_{\phi}+v d u^{*}=0
$$

where

$$
N_{\phi}\left(w_{1}, w_{2}\right)=\phi^{2}\left[w_{1}, w_{2}\right]+\left[\phi w_{1}, \phi w_{2}\right]-\phi\left[\phi w_{1}, w_{2}\right]-\phi\left[w_{1}, \phi w_{2}\right]
$$

for any $w_{1}, w_{2} \in \mathfrak{h}$. 
Here $d$ stands for Chevalley-Eilenberg exterior differential, cf. [8], being a linear version of the usual exterior differential. It is defined by

$$
d \omega(x, y)=-\omega([x, y])
$$

for $\omega \in \Lambda^{1} \mathfrak{g}^{*}$ and $x, y \in \mathfrak{g}$.

REMARK 2.10. A classical result, cf. [5], states that if $\mathfrak{g}$ admits a normal almost contact structure then $\mathfrak{g} \times \mathbb{R}$ admits a canonical complex structure. This is sometimes taken as a definition and the normality condition above becomes a theorem then.

If two algebras $\mathfrak{g}, \mathfrak{h}$ admit normal almost contact structures then $\mathfrak{g} \times \mathfrak{h}$ admits the canonical complex structure associated to these structures, which follows from Morimoto's result [14].

The lemma below guarantees the existence of a normal almost contact structure on the algebras satisfying certain structure conditions and the next lemma shows that in fact all of the other algebras from Theorem 2.4 fulfill these assumptions.

LEMma 2.11. Let $\mathfrak{g}=\operatorname{span}\left\{e_{1}, e_{2}, e_{3}\right\}$ be a Lie algebra such that $e_{1}$, and $e_{2}$ are the eigenvectors for the same real eigenvalue $\lambda$ or conjugated eigenvectors for a complex eigenvalue $a+$ bi of an adjoint endomorphism

$$
\left[\cdot, e_{3}\right]: \mathfrak{g} \rightarrow \mathfrak{g} .
$$

Then an almost contact structure $\left(\mathfrak{g}, \phi, e_{3}^{*}, e_{3}\right)$ defined by

$$
\begin{aligned}
p h i\left(e_{1}\right) & =e_{2}, \\
\phi\left(e_{2}\right) & =-e_{1}, \\
\phi\left(e_{3}\right) & =0,
\end{aligned}
$$

is normal.

Proof. We need to check the condition $N_{\phi}+\left(d e_{3}^{*}\right) e_{3}=0$. It is easy to see that

$$
\begin{aligned}
& N_{\phi}\left(e_{1}, e_{2}\right)+\left(d e_{3}^{*}\right)\left(e_{1}, e_{2}\right) e_{3}=\phi^{2}\left[e_{1}, e_{2}\right]+\left[e_{2},-e_{1}\right]-e_{3}^{*}\left(\left[e_{1}, e_{2}\right]\right) e_{3}=0, \\
& N_{\phi}\left(e_{1}, e_{3}\right)+\left(d e_{3}^{*}\right)\left(e_{1}, e_{3}\right) e_{3}=\left\{\begin{array}{l}
-\lambda e_{1}-\phi\left(\lambda e_{2}\right)=0 \\
-a e_{1}-b e_{2}-\phi\left(-b e_{1}+a e_{2}\right)=0
\end{array},\right. \\
& N_{\phi}\left(e_{2}, e_{3}\right)+\left(d e_{3}^{*}\right)\left(e_{2}, e_{3}\right) e_{3}=\left\{\begin{array}{l}
-\lambda e_{2}-\phi\left(-\lambda e_{1}\right)=0 \\
b e_{1}-a e_{2}-\phi\left(-a e_{1}-b e_{2}\right)=0
\end{array},\right.
\end{aligned}
$$

depending on whether we are considering the real eigenvalue or the complex one. 
Lemma 2.12. The Lie algebras $A_{3,1}, A_{3,2}, A_{3,3}, A_{3,4}, A_{3,7}^{\theta}$ for $\theta \geq 0$ and $A_{3,8}, A_{3,9}$ all admit a normal almost contact structure.

Proof. The bases given in Theorem 2.4 satisfy the assumptions of Lemma 2.11 .

REMARK 2.13. The Lie algebras from Lemma 2.12 are the only three dimensional Lie algebras admitting a normal almost contact structure. This follows from the mentioned result of Morimoto, cf. [14], that if two manifolds admit a normal almost contact structure then their product admits an integrable complex structure. In particular if any of $A_{3,5}^{\theta}$ or $A_{3,6}$ admits a normal almost contact structure then its product with itself admits complex structure, which contradicts Propositions 2.5, 2.6, 2.8.

We are ready to finish the section with the announced classification of the algebras of the form $\mathfrak{g}_{3} \times \mathfrak{h}_{3}$ which admit complex structures.

ThEOREM 2.14. The Lie algebra of the form $\mathfrak{g} \times \mathfrak{h}$ admits a complex structure if in the table below the symbol $\boldsymbol{\checkmark}$ is placed at the intersection of the raw corresponding to $\mathfrak{g}$ and the column corresponding to $\mathfrak{h}$; if it does not, then the symbol $\boldsymbol{X}$ is placed instead.

\begin{tabular}{|c|c|c|c|c|c|c|c|c|c|c|c|}
\hline$\overline{\mathfrak{g} \times \mathfrak{h}}$ & $A_{3,1}$ & $A_{3,2}$ & $A_{3,3}$ & $A_{3,4}$ & $A_{3,5}^{\theta}, \theta \neq-1$ & $A_{3,5}^{-1}$ & $A_{3,6}$ & $A_{3,7}^{\theta}, \theta>0$ & $A_{3,7}^{0}$ & $A_{3,8}$ & $A_{3,9}$ \\
\hline$A_{3,1}$ & $\checkmark$ & $\checkmark$ & $\checkmark$ & $\checkmark$ & $x$ & $x$ & $x$ & $\checkmark$ & $\checkmark$ & $\checkmark$ & $\checkmark$ \\
\hline$A_{3,2}$ & $\checkmark$ & $\checkmark$ & $\checkmark$ & $\sqrt{ }$ & $x$ & $x$ & $x$ & $\checkmark$ & $\checkmark$ & $\checkmark$ & $\checkmark$ \\
\hline$A_{3,3}$ & $\checkmark$ & $\checkmark$ & $\checkmark$ & $\checkmark$ & $x$ & $x$ & $x$ & $\checkmark$ & $\checkmark$ & $\checkmark$ & $\checkmark$ \\
\hline$A_{3,4}$ & $\checkmark$ & $\checkmark$ & $\checkmark$ & $\checkmark$ & $x$ & $x$ & $x$ & $\checkmark$ & $\checkmark$ & $\sqrt{2}$ & $\checkmark$ \\
\hline$A_{3,5}^{\theta}, \theta \neq-1$ & $x$ & $x$ & $x$ & $x$ & $x$ & $x$ & $x$ & $x$ & $x$ & $x$ & $x$ \\
\hline$A_{3,5}^{-1}$ & $x$ & $x$ & $x$ & $x$ & $x$ & $x$ & $x$ & $x$ & $\checkmark$ & $x$ & $x$ \\
\hline$A_{3,6}$ & $x$ & $x$ & $x$ & $x$ & $x$ & $x$ & $x$ & $x$ & $x$ & $x$ & $x$ \\
\hline$A_{3,7}^{\theta}, \theta>0$ & $\checkmark$ & $\checkmark$ & $\checkmark$ & $\checkmark$ & $x$ & $x$ & $x$ & $\checkmark$ & $\checkmark$ & $\checkmark$ & $\checkmark$ \\
\hline$A_{3,7}^{0}$ & $\sqrt{ }$ & $\checkmark$ & $\checkmark$ & $\sqrt{ }$ & $x$ & $\checkmark$ & $x$ & $\checkmark$ & $\checkmark$ & $\checkmark$ & $\checkmark$ \\
\hline$A_{3,8}$ & $\checkmark$ & $\checkmark$ & $\checkmark$ & $\sqrt{ }$ & $x$ & $x$ & $x$ & $\checkmark$ & $\checkmark$ & $\checkmark$ & $\checkmark$ \\
\hline$A_{3,9}$ & $\checkmark$ & $\checkmark$ & $\checkmark$ & $\checkmark$ & $x$ & $x$ & $x$ & $\checkmark$ & $\checkmark$ & $\checkmark$ & $\checkmark$ \\
\hline
\end{tabular}

REMARK 2.15. Results on the diagonal of the above table were analysed in $[\mathbf{9}]$.

3. Classification in dimension four. The classification of the Lie algebras up to dimension five has been known since Mubarakzjanov's paper [15]. In the theorem below we use the notation from [18] although we change the range of parameters in the fifth class in order to avoid double counting, since, as it can be easily checked, in this case if $\alpha=-1$ and $\beta>0$ then the substitution $e_{4}:=-e_{4}$ gives an isomorphism for the case $\alpha=-1$ and $\beta<0$.

TheOREM 3.1 ([18]). Every real four dimensional Lie algebra $\mathfrak{g}=$ $\operatorname{span}\left\{e_{1}, e_{2}, e_{3}, e_{4}\right\}$ is isomorphic to exactly one from the Lie algebras below. 


\section{Indecomposable}

$$
\begin{aligned}
A_{4,1}: & {\left[e_{2}, e_{4}\right]=e_{1},\left[e_{3}, e_{4}\right]=e_{2} } \\
A_{4,2}^{\alpha}: & {\left[e_{1}, e_{4}\right]=\alpha e_{1},\left[e_{2}, e_{4}\right]=e_{2},\left[e_{3}, e_{4}\right]=e_{2}+e_{3} } \\
& \text { for } \alpha \neq 0 \\
A_{4,3}: & {\left[e_{1}, e_{4}\right]=e_{1},\left[e_{3}, e_{4}\right]=e_{2} } \\
A_{4,4}: & {\left[e_{1}, e_{4}\right]=e_{1},\left[e_{2}, e_{4}\right]=e_{1}+e_{2},\left[e_{3}, e_{4}\right]=e_{2}+e_{3} } \\
A_{4,5}^{\alpha, \beta}: & {\left[e_{1}, e_{4}\right]=e_{1},\left[e_{2}, e_{4}\right]=\alpha e_{2},\left[e_{3}, e_{4}\right]=\beta e_{3} } \\
& \text { for } \alpha \beta \neq 0 \text { and }-1<\alpha \leq \beta \leq 1 \text { or }-1=\alpha \leq \beta<0 \\
A_{4,6}^{\alpha, \beta}: & {\left[e_{1}, e_{4}\right]=\alpha e_{1},\left[e_{2}, e_{4}\right]=\beta e_{2}-e_{3},\left[e_{3}, e_{4}\right]=e_{2}+\beta e_{3} } \\
& \text { for } \alpha \neq 0 \text { and } \beta \geq 0 \\
A_{4,7}: & {\left[e_{2}, e_{3}\right]=e_{1},\left[e_{1}, e_{4}\right]=2 e_{1},\left[e_{2}, e_{4}\right]=e_{2},\left[e_{3}, e_{4}\right]=e_{2}+e_{3} } \\
A_{4,8}: & {\left[e_{2}, e_{3}\right]=e_{1},\left[e_{2}, e_{4}\right]=e_{2},\left[e_{3}, e_{4}\right]=-e_{3} } \\
A_{4,9}^{\beta}: & {\left[e_{2}, e_{3}\right]=e_{1},\left[e_{1}, e_{4}\right]=(1+\beta) e_{1},\left[e_{2}, e_{4}\right]=e_{2},\left[e_{3}, e_{4}\right]=\beta e_{3} } \\
& \text { for }-1<\beta \leq 1 \\
A_{4,10}: & {\left[e_{2}, e_{3}\right]=e_{1},\left[e_{2}, e_{4}\right]=-e_{3},\left[e_{3}, e_{4}\right]=e_{2} } \\
A_{4,11}^{\alpha}: & {\left[e_{2}, e_{3}\right]=e_{1},\left[e_{1}, e_{4}\right]=2 \alpha e_{1},\left[e_{2}, e_{4}\right]=\alpha e_{2}-e_{3},\left[e_{3}, e_{4}\right]=e_{2}+\alpha e_{3} } \\
& \text { for } \alpha>0 \\
A_{4,12}: & {\left[e_{1}, e_{3}\right]=e_{1},\left[e_{2}, e_{3}\right]=e_{2},\left[e_{1}, e_{4}\right]=-e_{2},\left[e_{2}, e_{4}\right]=e_{1} }
\end{aligned}
$$

\section{Decomposable}

$\mathfrak{h} \times \mathbb{R}$ for any $\mathfrak{h}$ from Theorem 2.4

$\left[e_{1}, e_{2}\right]=e_{1},\left[e_{3}, e_{4}\right]=e_{3}$, i.e., $A_{2,2} \times A_{2,2}$ where $A_{2,2}$ is the only non-abelian two dimensional Lie algebra.

Combining the results from $[\mathbf{1 7}, \mathbf{2 3}]$ and noticing that the only non-solvable four dimensional Lie algebras are $A_{3,8} \times \mathbb{R}$ and $A_{3,9} \times \mathbb{R}$ (which admit a complex structure, because, as we have seen in the previous section, $A_{3,8}, A_{3,9}$ admit a normal almost contact structure), results in the classification for all Lie algebras in dimension four admitting complex structures.

Theorem $3.2([\mathbf{1 7}, \mathbf{2 3}])$. A four dimensional real Lie algebra $\mathfrak{g}$ admits a complex structure if and only if it is isomorphic to $A_{4,2}^{1}, A_{4,5}^{\alpha, \alpha}$ or $A_{4,5}^{\alpha, 1}$ for any $\alpha$ allowed by Theorem 3.1, $A_{4,6}^{\alpha, \beta}-A_{4,12}, \mathfrak{h} \times \mathbb{R}$ where $\mathfrak{h}$ is any Lie algebra from Lemma 2.12 or $A_{2,2} \times A_{2,2}$. 
4. Complex structures on Lie algebras of the form $\mathfrak{g}_{4} \times \mathbb{R}^{2}$ and $\mathfrak{g}_{4} \times A_{2,2}$. In this section we continue the classification in dimension six for decomposable algebras with a four dimensional summand. Since in Section 2 we have covered with all three dimensional algebras and because every two dimensional Lie algebra admits a complex structure, we may assume that the four dimensional summand is indecomposable. Since any almost complex structure on two dimensional Lie algebra is integrable and thanks to Theorem 3.2 , we only have to deal with algebras $\mathfrak{g} \times \mathbb{R}^{2}$ and $\mathfrak{g} \times A_{2,2}$ where $\mathfrak{g}$ is one of $A_{4,1}, A_{4,2}^{\alpha}$ where $\alpha \neq 1, A_{4,3}, A_{4,4}$ and $A_{4,5}^{\alpha, \beta}$ for $-1<\alpha<\beta<1$ or $-1=\alpha<\beta<0$. This section is quite computable since most proofs reduce to examining zero torsion endomorphisms on the four dimensional summand. This in turn reduces to writing down torsion conditions and obtaining the associated system of polynomial equations. We treat each case separately because the systems obtained differ substantially from one another, in order to make possible future references easier and to make the section better structured. As before, we start with the most rigid cases, i.e., with those $\mathfrak{g}$ which do not admit even a zero torsion endomorphism.

Proposition 4.1. There are no zero torsion endomorphisms on Lie algebras $A_{4,2}^{\alpha}$ for $\alpha \neq 1$.

Proof. For the purpose of the proof let us denote $\omega=1-\alpha$. Suppose there is a zero torsion endomorphism $\mathcal{I}$ on $A_{4,2}^{\alpha}$ for $\alpha \neq 1$ given in the basis from Theorem 3.1 by the matrix below

$$
\left[\begin{array}{cccc}
a & A & k & K \\
b & B & l & L \\
c & C & m & M \\
d & D & n & N
\end{array}\right] .
$$

We write down the torsion conditions for $\mathcal{I}$.

$$
\begin{aligned}
{[\mathcal{I}, \mathcal{I}]\left(e_{1}, e_{2}\right)=} & -\left[e_{1}, e_{2}\right]+\left[\sum a e_{i}, \sum A e_{i}\right]-\mathcal{I}\left[\sum a e_{i}, e_{2}\right]-\mathcal{I}\left[e_{1}, \sum A e_{i}\right] \\
= & a D \alpha e_{1}+b D e_{2}+c D e_{2}+c D e_{3}-d A \alpha e_{1}-d B e_{2}-d C e_{2} \\
& -d C e_{3}+\mathcal{I}\left(d e_{2}\right)-\mathcal{I}\left(D \alpha e_{1}\right) \\
= & a D \alpha e_{1}+b D e_{2}+c D e_{2}+c D e_{3}-d A \alpha e_{1}-d B e_{2}-d C e_{2} \\
& -d C e_{3}+d\left(\sum A e_{i}\right)-D \alpha\left(\sum a e_{i}\right) \\
= & (a D \alpha-d A \alpha+d A-D a \alpha) e_{1} \\
& +(b D+c D-d B-d C+d B-D b \alpha) e_{2} \\
& +(c D-d C+d C-D c \alpha) e_{3}+(d D-D d \alpha) e_{4}
\end{aligned}
$$




$$
\begin{aligned}
{[\mathcal{I}, \mathcal{I}]\left(e_{1}, e_{3}\right)=} & -\left[e_{1}, e_{3}\right]+\left[\sum a e_{i}, \sum k e_{i}\right]-\mathcal{I}\left[\sum a e_{i}, e_{3}\right]-\mathcal{I}\left[e_{1}, \sum k e_{i}\right] \\
= & a n \alpha e_{1}+b n e_{2}+c n e_{2}+c n e_{3}-d k \alpha e_{1}-d l e_{2}-d m e_{2}-d m e_{3} \\
& +\mathcal{I}\left(d e_{2}+d e_{3}\right)-\mathcal{I}\left(n \alpha e_{1}\right) \\
= & a n \alpha e_{1}+b n e_{2}+c n e_{2}+c n e_{3}-d k \alpha e_{1}-d l e_{2}-d m e_{2}-d m e_{3} \\
& +d\left(\sum A e_{i}\right)+d\left(\sum k e_{i}\right)-n \alpha\left(\sum a e_{i}\right) \\
= & (a n \alpha-d k \alpha+d A+d k-n a \alpha) e_{1} \\
& +(b n+c n-d l-d m+d B+d l-n b \alpha) e_{2} \\
& +(c n-d m+d C+d m-n c \alpha) e_{3}+(d D+d n-n d \alpha) e_{4} \\
{[\mathcal{I}, \mathcal{I}]\left(e_{1}, e_{4}\right)=} & -\left[e_{1}, e_{4}\right]+\left[\sum a e_{i}, \sum K e_{i}\right]-\mathcal{I}\left[\sum a e_{i}, e_{4}\right]-\mathcal{I}\left[e_{1}, \sum K e_{i}\right] \\
= & -\alpha e_{1}+a N \alpha e_{1}+b N e_{2}+c N e_{2}+c N e_{3}-d K \alpha e_{1}-d L e_{2} \\
& -d M e_{2}-d M e_{3}-\mathcal{I}\left(a \alpha e_{1}+b e_{2}+c e_{2}+c e_{3}\right)-\mathcal{I}\left(N \alpha e_{1}\right) \\
= & -\alpha e_{1}+a N \alpha e_{1}+b N e_{2}+c N e_{2}+c N e_{3}-d K \alpha e_{1}-d L e_{2} \\
& -d M e_{2}-d M e_{3}-a \alpha\left(\sum a e_{i}\right)-(b+c)\left(\sum A e_{i}\right) \\
& -c\left(\sum k e_{i}\right)-N \alpha\left(\sum a e_{i}\right) \\
= & \left(-\alpha+a N \alpha-d K \alpha-a^{2} \alpha-b A-c A-c k-N a \alpha\right) e_{1} \\
& +(b N+c N-d L-d M-a b \alpha-b B-c B-c l-N b \alpha) e_{2} \\
& +(c N-d M-a c \alpha-b C-c C-c m-N c \alpha) e_{3} \\
& +(-a d \alpha-b D-c D-c n-N d \alpha) e_{4}
\end{aligned}
$$$$
[\mathcal{I}, \mathcal{I}]\left(e_{2}, e_{3}\right)=-\left[e_{2}, e_{3}\right]+\left[\sum A e_{i}, \sum k e_{i}\right]-\mathcal{I}\left[\sum A e_{i}, e_{3}\right]-\mathcal{I}\left[e_{2}, \sum k e_{i}\right]
$$$$
=A n \alpha e_{1}+B_{n e}+C n e_{2}+C n e_{3}-D k \alpha e_{1}-D l e_{2}-D m e_{2}
$$$$
-D \mathrm{me}_{3}+\mathcal{I}\left(D e_{2}+D e_{3}\right)-\mathcal{I}\left(n e_{2}\right)
$$$$
=\text { Ance }_{1}+\text { Bne }_{2}+C n e_{2}+C n e_{3}-D k \alpha e_{1}-D l e_{2}-D m e_{2}
$$$$
-D m e_{3}+D\left(\sum A e_{i}\right)+D\left(\sum k e_{i}\right)-n\left(\sum A e_{i}\right)
$$$$
=(A n \alpha-D k \alpha+D A+D k-n A) e_{1}
$$$$
+(B n+C n-D l-D m+D B+D l-n B) e_{2}
$$$$
+(C n-D m+D C+D m-n C) e_{3}+\left(D^{2}+D n-n D\right) e_{4}
$$

$$
\begin{aligned}
{[\mathcal{I}, \mathcal{I}]\left(e_{2}, e_{4}\right)=} & -\left[e_{2}, e_{4}\right]+\left[\sum A e_{i}, \sum K e_{i}\right]-\mathcal{I}\left[\sum A e_{i}, e_{4}\right]-\mathcal{I}\left[e_{2}, \sum K e_{i}\right] \\
= & -e_{2}+A N \alpha e_{1}+B N e_{2}+C N e_{2}+C N e_{3}-D K \alpha e_{1}-D L e_{2} \\
& -D M e_{2}-D M e_{3}-\mathcal{I}\left(A \alpha e_{1}+B e_{2}+C e_{2}+C e_{3}\right)-\mathcal{I}\left(N e_{2}\right)
\end{aligned}
$$




$$
\begin{aligned}
= & -e_{2}+A N \alpha e_{1}+B N e_{2}+C N e_{2}+C N e_{3}-D K \alpha e_{1}-D L e_{2} \\
& -D M e_{2}-D M e_{3}-A \alpha\left(\sum a e_{i}\right)-(B+C)\left(\sum A e_{i}\right) \\
& -C\left(\sum k e_{i}\right)-N\left(\sum A e_{i}\right) \\
= & (A N \alpha-D K \alpha-A a \alpha-B A-C A-C k-N A) e_{1} \\
& +\left(-1+B N+C N-D L-D M-A b \alpha-B^{2}-C B-C l-N B\right) e_{2} \\
& +\left(C N-D M-A c \alpha-B C-C^{2}-C m-N C\right) e_{3} \\
& +(-A d \alpha-B D-C D-C n-N D) e_{4}
\end{aligned}
$$

$$
\begin{aligned}
{[\mathcal{I}, \mathcal{I}]\left(e_{3}, e_{4}\right)=} & -\left[e_{3}, e_{4}\right]+\left[\sum k e_{i}, \sum K e_{i}\right]-\mathcal{I}\left[\sum k e_{i}, e_{4}\right]-\mathcal{I}\left[e_{3}, \sum K e_{i}\right] \\
= & -e_{2}-e_{3}+k N \alpha e_{1}+l N e_{2}+m N e_{2}+m N e_{3}-n K \alpha e_{1}-n L e_{2} \\
& -n M e_{2}-n M e_{3}-\mathcal{I}\left(k \alpha e_{1}+l e_{2}+m e_{2}+m e_{3}\right)-\mathcal{I}\left(N e_{2}+N e_{3}\right) \\
= & -e_{2}-e_{3}+k N \alpha e_{1}+l N e_{2}+m N e_{2}+m N e_{3}-n K \alpha e_{1}-n L e_{2} \\
& -n M e_{2}-n M e_{3}-k \alpha\left(\sum a e_{i}\right)-(l+m)\left(\sum A e_{i}\right) \\
& -m\left(\sum k e_{i}\right)-N\left(\sum A e_{i}\right)-N\left(\sum k e_{i}\right) \\
= & (k N \alpha-n K \alpha-k a \alpha-l A-m A-m k-N A-N k) e_{1} \\
& +(-1+l N+m N-n L-n M-k b \alpha-l B-m B-m l-N B-N l) e_{2} \\
& +\left(-1+m N-n M-k c \alpha-l C-m C-m^{2}-N C-N m\right) e_{3} \\
& +(-k d \alpha-l D-m D-m n-N D-N n) e_{4}
\end{aligned}
$$

Because $\alpha \neq 1$ this gives the following system of equations

(I)

$$
\left\{\begin{array}{l}
D=d C=d A=d k=d n=c n=C n=A n=0 \\
\omega b n-d m+d B=0 \\
-\alpha-d K \alpha-a^{2} \alpha-b A-c A-c k=0 \\
\omega b N+c N-d L-d M-a b \alpha-b B-c B-c l=0 \\
\omega c N-d M-a c \alpha-b C-c C-c m=0 \\
a d+N d=0 \\
-\omega A N-A a \alpha-B A-C A-C k=0 \\
-1+C N-A b \alpha-B^{2}-C B-C l=0 \\
-A c \alpha-B C-C^{2}-C m=0 \\
-\omega k N-n K \alpha-k a \alpha-l A-m A-m k-N A=0 \\
-1+m N-n L-n M-k b \alpha-l B-m B-m l-N B=0 \\
-1-n M-k c \alpha-l C-m C-m^{2}-N C=0 \\
-k d \alpha-m n-N n=0
\end{array}\right.
$$


Notice that $d \neq 0$ or $n \neq 0$ implies $C=A=0$, which contradicts the eighth equation. Consequently, the system above gives the following reduced system by considering only some equations and rewriting them

$$
\left\{\begin{array}{l}
\omega c N-a c \alpha-b C-c C-c m=0 \\
\omega A N+a A \alpha+B A+C A+C k=0 \\
c A \alpha+B C+C^{2}+C m=0 \\
\alpha+a^{2} \alpha+b A+c A+c k=0 \\
1+k c \alpha+l C+m C+m^{2}+C N=0 \\
1-C N+b A \alpha+B^{2}+C B+C l=0
\end{array} .\right.
$$

Note that from the third equation $C=0$ would imply $c=0$ or $A=0$, which is impossible because of the last two equations. Applying the third equation in the first and the second ones gives after multiplication

$$
\begin{aligned}
\alpha b A & =\frac{\alpha c A}{C}(\omega N-a \alpha-C-m)=-(C+B+m)(\omega N-a \alpha-C-m), \\
\alpha c k & =-\frac{\alpha c A}{C}(\omega N+a \alpha+B+C)=(C+B+m)(\omega N+a \alpha+B+C) .
\end{aligned}
$$

Thus the second and the third equations give

$$
\alpha(b A+c k+c A)=(B+C+m)(2 a \alpha+C+B+m) .
$$

Taking into account the fourth equation of $(I I)$ we obtain

$$
-\alpha^{2}-(a \alpha)^{2}=(B+C+m)(2 a \alpha+C+B+m)
$$

or just

$$
\alpha^{2}+(B+C+m+a \alpha)^{2}=0
$$

an obvious contradiction since $\alpha \neq 0$.

Proposition 4.2. There is no zero torsion endomorphism on the Lie algebra $A_{4,3}$.

Proof. Suppose there is a zero torsion endomorphism $\mathcal{I}$ on $A_{4,3}$ given in the basis from Theorem 3.1 by the matrix defined in Proposition 4.1. The torsion conditions are displayed below.

$$
\begin{aligned}
{[\mathcal{I}, \mathcal{I}]\left(e_{1}, e_{2}\right) } & =-\left[e_{1}, e_{2}\right]+\left[\sum a e_{i}, \sum A e_{i}\right]-\mathcal{I}\left[\sum a e_{i}, e_{2}\right]-\mathcal{I}\left[e_{1}, \sum A e_{i}\right] \\
& =a D e_{1}+c D e_{2}-d A e_{1}-d C e_{2}-\mathcal{I}\left(D e_{1}\right) \\
& =a D e_{1}+c D e_{2}-d A e_{1}-d C e_{2}-D\left(\sum a e_{i}\right) \\
& =(a D-d A-D a) e_{1}+(c D-d C-D b) e_{2}+(-D c) e_{3}+(-D d) e_{4}
\end{aligned}
$$




$$
\begin{aligned}
{[\mathcal{I}, \mathcal{I}]\left(e_{1}, e_{3}\right)=} & -\left[e_{1}, e_{3}\right]+\left[\sum a e_{i}, \sum k e_{i}\right]-\mathcal{I}\left[\sum a e_{i}, e_{3}\right]-\mathcal{I}\left[e_{1}, \sum k e_{i}\right] \\
= & a n e_{1}+c n e_{2}-d k e_{1}-d m e_{2}+\mathcal{I}\left(d e_{2}\right)-\mathcal{I}\left(n e_{1}\right) \\
= & a n e_{1}+c n e_{2}-d k e_{1}-d m e_{2}+d\left(\sum A e_{i}\right)-n\left(\sum a e_{i}\right) \\
= & (a n-d k+d A-n a) e_{1}+(c n-d m+d B-n b) e_{2} \\
& \quad+(d C-n c) e_{3}+(d D-n d) e_{4} \\
{[\mathcal{I}, \mathcal{I}]\left(e_{1}, e_{4}\right)=- } & {\left[e_{1}, e_{4}\right]+\left[\sum a e_{i}, \sum K e_{i}\right]-\mathcal{I}\left[\sum a e_{i}, e_{4}\right]-\mathcal{I}\left[e_{1}, \sum K e_{i}\right] } \\
= & -e_{1}+a N e_{1}+c N e_{2}-d K e_{1}-d M e_{2}-\mathcal{I}\left(a e_{1}+c e_{2}\right)-\mathcal{I}\left(N e_{1}\right) \\
= & -e_{1}+a N e_{1}+c N e_{2}-d K e_{1}-d M e_{2} \\
& -a\left(\sum a e_{i}\right)-c\left(\sum A e_{i}\right)-N\left(\sum a e_{i}\right) \\
= & \left(-1+a N-d K-a^{2}-c A-N a\right) e_{1} \\
& +(c N-d M-a b-c B-N b) e_{2} \\
& +(-a c-c C-N c) e_{3}+(-a d-c D-N d) e_{4}
\end{aligned}
$$

$$
\begin{aligned}
{[\mathcal{I}, \mathcal{I}]\left(e_{2}, e_{3}\right) } & =-\left[e_{2}, e_{3}\right]+\left[\sum A e_{i}, \sum k e_{i}\right]-\mathcal{I}\left[\sum A e_{i}, e_{3}\right]-\mathcal{I}\left[e_{2}, \sum k e_{i}\right] \\
& =A n e_{1}+C n e_{2}-D k e_{1}-D m e_{2}+\mathcal{I}\left(D e_{2}\right) \\
& =A n e_{1}+C n e_{2}-D k e_{1}-D m e_{2}+D\left(\sum A e_{i}\right) \\
& =(A n-D k+D A) e_{1}+(C n-D m+D B) e_{2}+(D C) e_{3}+\left(D^{2}\right) e_{4}
\end{aligned}
$$

$$
\begin{aligned}
{[\mathcal{I}, \mathcal{I}]\left(e_{2}, e_{4}\right)=} & -\left[e_{2}, e_{4}\right]+\left[\sum A e_{i}, \sum K e_{i}\right]-\mathcal{I}\left[\sum A e_{i}, e_{4}\right]-\mathcal{I}\left[e_{2}, \sum K e_{i}\right] \\
= & A N e_{1}+C N e_{2}-D K e_{1}-D M e_{2}-\mathcal{I}\left(A e_{1}+C e_{2}\right) \\
= & A N e_{1}+C N e_{2}-D K e_{1}-D M e_{2}-A\left(\sum a e_{i}\right)-C\left(\sum A e_{i}\right) \\
= & (A N-D K-A a-C A) e_{1}+(C N-D M-A b-C B) e_{2} \\
& +\left(-A c-C^{2}\right) e_{3}+(-A d-C D) e_{4}
\end{aligned}
$$

$$
\begin{aligned}
{[\mathcal{I}, \mathcal{I}]\left(e_{3}, e_{4}\right)=} & -\left[e_{3}, e_{4}\right]+\left[\sum k e_{i}, \sum K e_{i}\right]-\mathcal{I}\left[\sum k e_{i}, e_{4}\right]-\mathcal{I}\left[e_{3}, \sum K e_{i}\right] \\
= & -e_{2}+k N e_{1}+m N e_{2}-n K e_{1}-n M e_{2}-\mathcal{I}\left(k e_{1}+m e_{2}\right)-\mathcal{I}\left(N e_{2}\right) \\
= & -e_{2}+k N e_{1}+m N e_{2}-n K e_{1}-n M e_{2} \\
& -k\left(\sum a e_{i}\right)-m\left(\sum A e_{i}\right)-N\left(\sum A e_{i}\right) \\
= & (k N-n K-k a-m A-N A) e_{1} \\
& +(-1+m N-n M-k b-m B-N B) e_{2} \\
& +(-k c-m C-N C) e_{3}+(-k d-m D-N D) e_{4}
\end{aligned}
$$


We merge them into the following system

$$
\left\{\begin{array}{l}
D=d A=d C=d k=d n=n c=n A=n C=0 \\
C^{2}+A c=0 \\
d B-d m-n b=0 \\
a d+N d=0 \\
c(a+C+N)=0 \\
A(N-a-C)=0 \\
C N-A b-C B=0 \\
k c+C m+N C=0 \\
c N-d M-a b-c B-N b=0 \\
k N-n K-k a-m A-N A=0 \\
1+d K+a^{2}+c A=0 \\
-1+m N-n M-k b-m B-N B=0
\end{array}\right.
$$

Notice that $d \neq 0$ implies $n=k=0$, which in turn, from the third equation, gives $m=B$. From the last equation we have $-1-m^{2}=0$. This means $d=0$. From the last but one equation we deduce that $A \neq 0$ and $c \neq 0$. Combining the fifth and the sixth equations we obtain $a=-C$. Applying this and $A c=-C^{2}$ in the last but one equation results in $1+C^{2}-C^{2}=0$, a contradiction.

Proposition 4.3. There is no zero torsion endomorphism on the Lie algebra $A_{4,4}$.

Proof. The proof goes as in the previous proposition. Suppose there is a zero torsion endomorphism $\mathcal{I}$ on $A_{4,4}$ given by the matrix as before, then

$$
\begin{aligned}
{[\mathcal{I}, \mathcal{I}]\left(e_{1}, e_{2}\right)=} & -\left[e_{1}, e_{2}\right]+\left[\sum a e_{i}, \sum A e_{i}\right]-\mathcal{I}\left[\sum a e_{i}, e_{2}\right]-\mathcal{I}\left[e_{1}, \sum A e_{i}\right] \\
= & a D e_{1}+b D e_{1}+b D e_{2}+c D e_{2}+c D e_{3}-d A e_{1}-d B e_{1}-d B e_{2} \\
& -d C e_{2}-d C e_{3}+\mathcal{I}\left(d e_{1}+d e_{2}\right)-\mathcal{I}\left(D e_{1}\right) \\
= & a D e_{1}+b D e_{1}+b D e_{2}+c D e_{2}+c D e_{3}-d A e_{1}-d B e_{1}-d B e_{2} \\
& -d C e_{2}-d C e_{3}+d\left(\sum a e_{i}\right)+d\left(\sum A e_{i}\right)-D\left(\sum a e_{i}\right) \\
= & (a D+b D-d A-d B+d a+d A-D a) e_{1} \\
& +(b D+c D-d B-d C+d b+d B-D b) e_{2} \\
& +(c D-d C+d c+d C-D c) e_{3}+\left(d^{2}+d D-D d\right) e_{4}
\end{aligned}
$$




$$
\begin{aligned}
{[\mathcal{I}, \mathcal{I}]\left(e_{1}, e_{3}\right)=} & -\left[e_{1}, e_{3}\right]+\left[\sum a e_{i}, \sum k e_{i}\right]-\mathcal{I}\left[\sum a e_{i}, e_{3}\right]-\mathcal{I}\left[e_{1}, \sum k e_{i}\right] \\
= & a n e_{1}+b n e_{1}+b n e_{2}+c n e_{2}+c n e_{3}-d k e_{1}-d l e_{1}-d l e_{2} \\
& -d m e_{2}-d m e_{3}+\mathcal{I}\left(d e_{2}+d e_{3}\right)-\mathcal{I}\left(n e_{1}\right) \\
= & a n e_{1}+b n e_{1}+b n e_{2}+c n e_{2}+c n e_{3}-d k e_{1}-d l e_{1}-d l e_{2} \\
& -d m e_{2}-d m e_{3}+d\left(\sum A e_{i}\right)+d\left(\sum k e_{i}\right)-n\left(\sum a e_{i}\right) \\
= & (a n+b n-d k-d l+d A+d k-n a) e_{1} \\
& +(b n+c n-d l-d m+d B+d l-n b) e_{2} \\
& +(c n-d m+d C+d m-n c) e_{3}+(d D+d n-n d) e_{4}
\end{aligned}
$$

$$
\begin{aligned}
{[\mathcal{I}, \mathcal{I}]\left(e_{1}, e_{4}\right)=} & -\left[e_{1}, e_{4}\right]+\left[\sum a e_{i}, \sum K e_{i}\right]-\mathcal{I}\left[\sum a e_{i}, e_{4}\right]-\mathcal{I}\left[e_{1}, \sum K e_{i}\right] \\
= & -e_{1}+a N e_{1}+b N e_{1}+b N e_{2}+c N e_{2}+c N e_{3}-d K e_{1}-d L e_{1} \\
& -d L e_{2}-d M e_{2}-d M e_{3}-\mathcal{I}\left(a e_{1}+b e_{1}+b e_{2}+c e_{2}+c e_{3}\right)-\mathcal{I}\left(N e_{1}\right) \\
= & a N e_{1}+b N e_{1}+b N e_{2}+c N e_{2}+c N e_{3}-d K e_{1}-d L e_{1}-d L e_{2} \\
& -d M e_{2}-d M e_{3}-(a+b)\left(\sum a e_{i}\right)-(b+c)\left(\sum A e_{i}\right)-c\left(\sum k e_{i}\right) \\
& -N\left(\sum a e_{i}\right) \\
= & \left(-1+a N+b N-d K-d L-a^{2}-a b-b A-c A-c k-N a\right) e_{1} \\
& +\left(b N+c N-d L-d M-a b-b^{2}-b B-c B-c l-N b\right) e_{2} \\
& +(c N-d M-a c-b c-b C-c C-c m-N c) e_{3} \\
& +(-a d-b d-b D-c D-c n-N d) e_{4}
\end{aligned}
$$

$$
\begin{aligned}
{[\mathcal{I}, \mathcal{I}]\left(e_{2}, e_{3}\right)=} & -\left[e_{2}, e_{3}\right]+\left[\sum A e_{i}, \sum k e_{i}\right]-\mathcal{I}\left[\sum A e_{i}, e_{3}\right]-\mathcal{I}\left[e_{2}, \sum k e_{i}\right] \\
= & A n e_{1}+B n e_{1}+B n e_{2}+C n e_{2}+C n e_{3}-D k e_{1}-D l e_{1}-D l e_{2} \\
& -D m e_{2}-D m e_{3}+\mathcal{I}\left(D e_{2}+D e_{3}\right)-\mathcal{I}\left(n e_{1}+n e_{2}\right) \\
= & A n e_{1}+B n e_{1}+B n e_{2}+C n e_{2}+C n e_{3}-D k e_{1}-D l e_{1}-D l e_{2} \\
& -D m e_{2}-D m e_{3}+D\left(\sum A e_{i}\right)+D\left(\sum k e_{i}\right)-n\left(\sum a e_{i}\right)-n\left(\sum A e_{i}\right) \\
= & (A n+B n-D k-D l+D A+D k-n a-n A) e_{1} \\
& +(B n+C n-D l-D m+D B+D l-n b-n B) e_{2} \\
& +(C n-D m+D C+D m-n c-n C) e_{3} \\
& +\left(D^{2}+D n-n d-n D\right) e_{4}
\end{aligned}
$$




$$
\begin{aligned}
{[\mathcal{I}, \mathcal{I}]\left(e_{2}, e_{4}\right)=} & -\left[e_{2}, e_{4}\right]+\left[\sum A e_{i}, \sum K e_{i}\right]-\mathcal{I}\left[\sum A e_{i}, e_{4}\right]-\mathcal{I}\left[e_{2}, \sum K e_{i}\right] \\
= & -e_{1}-e_{2}+A N e_{1}+B N e_{1}+B N e_{2}+C N e_{2}+C N e_{3}-D K e_{1} \\
& -D L e_{1}-D L e_{2}-D M e_{2}-D M e_{3} \\
& -\mathcal{I}\left(A e_{1}+B e_{1}+B e_{2}+C e_{2}+C e_{3}\right)-\mathcal{I}\left(N e_{1}+N e_{2}\right) \\
= & -e_{1}-e_{2}+A N e_{1}+B N e_{1}+B N e_{2}+C N e_{2}+C N e_{3}-D K e_{1} \\
& -D L e_{1}-D L e_{2}-D M e_{2}-D M e_{3}-(A+B)\left(\sum a e_{i}\right) \\
& -(B+C)\left(\sum A e_{i}\right)-C\left(\sum k e_{i}\right)-N\left(\sum a e_{i}\right)-N\left(\sum A e_{i}\right) \\
= & (-1+A N+B N-D K-D L-A a-B a \\
& \quad-B A-C A-C k-N a-N A) e_{1} \\
& +(-1+B N+C N-D L-D M-A b-B b \\
& -B 2-C B-C l-N b-N B) e_{2} \\
& +\left(C N-D M-A c-B c-B C-C^{2}-C m-N c-N C\right) e_{3} \\
& +(-A d-B d-B D-C D-C n-N d-N D) e_{4}
\end{aligned}
$$

$$
\begin{aligned}
{[\mathcal{I}, \mathcal{I}]\left(e_{3}, e_{4}\right)=} & -\left[e_{3}, e_{4}\right]+\left[\sum k e_{i}, \sum K e_{i}\right]-\mathcal{I}\left[\sum k e_{i}, e_{4}\right]-\mathcal{I}\left[e_{3}, \sum K e_{i}\right] \\
= & -e_{2}-e_{3}+k N e_{1}+l N e_{1}+l N e_{2}+m N e_{2}+m N e_{3}-n K e_{1} \\
& -n L e_{1}-n L e_{2}-n M e_{2}-n M e_{3} \\
& -\mathcal{I}\left(k e_{1}+l e_{1}+l e_{2}+m e_{2}+m e_{3}\right)-\mathcal{I}\left(N e_{2}+N e_{3}\right) \\
= & -e_{2}-e_{3}+k N e_{1}+l N e_{1}+l N e_{2}+m N e_{2}+m N e_{3}-n K e_{1} \\
& -n L e_{1}-n L e_{2}-n M e_{2}-n M e_{3}-(k+l)\left(\sum a e_{i}\right) \\
& -(l+m)\left(\sum A e_{i}\right)-m\left(\sum k e_{i}\right)-N\left(\sum A e_{i}\right)-N\left(\sum k e_{i}\right) \\
= & (k N+l N-n K-n L-k a-l a \\
& \quad-l A-m A-m k-N A-N k) e_{1} \\
& +(-1+l N+m N-n L-n M-k b \\
& \quad-l b-l B-m B-m l-N B-N l) e_{2} \\
& +\left(-1+m N-n M-k c-l c-l C-m C-m^{2}-N C-N m\right) e_{3} \\
& +(-k d-l d-l D-m D-m n-N D-N n) e_{4} .
\end{aligned}
$$


After cancellations and easy manipulations we obtain

$$
\left\{\begin{array}{l}
d=D=b n=c n=C n=0 \\
B n-n a=m n+N n=0 \\
a c+b c+b C+c C+c m=0 \\
-1+b N-a^{2}-a b-b A-c A-c k=0 \\
c N-a b-b^{2}-b B-c B-c l=0 \\
-1+B N-A a-B a-B A-C A-C k-N a=0 \\
-1+C N-A b-B b-B^{2}-C B-C l-N b=0 \\
A c+B c+B C+C^{2}+C m+N c=0 \\
l N-n K-n L-k a-l a-l A-m A-m k-N A=0 \\
-1+m N-n L-n M-k b-l b-l B-m B-m l-N B=0 \\
-1-n M-k c-l c-l C-m C-m^{2}-N C=0
\end{array}\right.
$$

One easily observes that $n \neq 0$ implies $b=c=0$, which gives $1+a^{2}=0$ from the fourth equation, which is an absurd. Consequently, $n=0$ and we obtain the following reduced system by considering only some of the equations

$$
\left\{\begin{array}{l}
-b C=c(a+b+C+m) \\
c(N-B-l)=b(a+b+B) \\
c(A+B+N)=-C(C+B+m) \\
-1+b N-a^{2}-a b-b A-c A-c k=0 \\
-1-k c-l c-l C-m C-m^{2}-N C=0 \\
-1-B^{2}+C(-B-l+N)-b(N+A+B)=0
\end{array}\right.
$$

From the first equation, $c=0$ gives $b C=0$, which is impossible because of the fourth and the fifth equations; consequently $c \neq 0$. Finally, using the first, second and third equations we can rewrite the last equation as

$$
\begin{aligned}
0 & =-1-B^{2}-(a+b+C+m)(a+b+B)-(a+b+C+m)(C+B+m) \\
& =-1-B^{2}-(a+b+C+m)((a+b+C+m)+2 B) \\
& =-1-(a+b+C+m+B)^{2}
\end{aligned}
$$

which gives a desired contradiction.

Proposition 4.4. There is no zero torsion endomorphism on Lie algebras $A_{4,5}^{\alpha, \beta}$ for $-1<\alpha<\beta<1$ and $-1=\alpha<\beta<0$.

Proof. Suppose there is a zero torsion endomorphism $\mathcal{I}$ on some of these algebras with the matrix representation as before. Let us write down the torsion conditions. 


$$
\begin{aligned}
{[\mathcal{I}, \mathcal{I}]\left(e_{1}, e_{2}\right)=} & -\left[e_{1}, e_{2}\right]+\left[\sum a e_{i}, \sum A e_{i}\right]-\mathcal{I}\left[\sum a e_{i}, e_{2}\right]-\mathcal{I}\left[e_{1}, \sum A e_{i}\right] \\
= & a D e_{1}+b \alpha D e_{2}+c \beta D e_{3}-d A e_{1}-d B \alpha e_{2}-d \beta C e_{3} \\
& +\mathcal{I}\left(d \alpha e_{2}\right)-\mathcal{I}\left(D e_{1}\right) \\
= & a D e_{1}+b \alpha D e_{2}+c \beta D e_{3}-d A e_{1}-d B \alpha e_{2}-d \beta C e_{3} \\
& +\alpha d\left(\sum A e_{i}\right)-D\left(\sum a e_{i}\right) \\
= & (a D-d A+\alpha d A-D a) e_{1}+(\alpha b D-\alpha d B+\alpha d B-D b) e_{2} \\
& +(\beta c D-\beta d C+\alpha d C-D c) e_{3}+(\alpha d D-D d) e_{4}
\end{aligned}
$$

$$
\begin{aligned}
{[\mathcal{I}, \mathcal{I}]\left(e_{1}, e_{3}\right)=} & -\left[e_{1}, e_{3}\right]+\left[\sum a e_{i}, \sum k e_{i}\right]-\mathcal{I}\left[\sum a e_{i}, e_{3}\right]-\mathcal{I}\left[e_{1}, \sum k e_{i}\right] \\
= & a_{n} e_{1}+b \alpha n e_{2}+c \beta n e_{3}-d k e_{1}-d l \alpha e_{2}-d \beta m e_{3} \\
& +\mathcal{I}\left(d \beta e_{3}\right)-\mathcal{I}\left(n e_{1}\right) \\
= & a_{n e_{1}}+b \alpha n e_{2}+c \beta n e_{3}-d k e_{1}-d l \alpha e_{2}-d \beta m e_{3} \\
& +\beta d\left(\sum k e_{i}\right)-n\left(\sum a e_{i}\right) \\
= & (a n-d k+\beta d k-n a) e_{1}+(\alpha b n-\alpha d l+\beta d l-n b) e_{2} \\
& +(\beta c n-\beta d m+\beta d m-n c) e_{3}+(\beta d n-d n) e_{4}
\end{aligned}
$$

$$
\begin{aligned}
{[\mathcal{I}, \mathcal{I}]\left(e_{1}, e_{4}\right)=} & -\left[e_{1}, e_{4}\right]+\left[\sum a e_{i}, \sum K e_{i}\right]-\mathcal{I}\left[\sum a e_{i}, e_{4}\right]-\mathcal{I}\left[e_{1}, \sum K e_{i}\right] \\
= & -e_{1}+a N e_{1}+b \alpha N e_{2}+c \beta N e_{3}-d K e_{1}-d \alpha L e_{2}-d \beta M e_{3} \\
& -\mathcal{I}\left(a e_{1}+\alpha b e_{2}+\beta c e_{3}\right)-\mathcal{I}\left(N e_{1}\right) \\
= & -e_{1}+a N e_{1}+b \alpha N e_{2}+c \beta N e_{3}-d K e_{1}-d \alpha L e_{2}-d \beta M e_{3} \\
& -a\left(\sum a e_{i}\right)-\alpha b\left(\sum A e_{i}\right)-\beta c\left(\sum k e_{i}\right)-N\left(\sum a e_{i}\right) \\
= & \left(-1+a N-d K-a^{2}-\alpha b A-\beta c k-N a\right) e_{1} \\
& +(\alpha b N-\alpha d L-a b-\alpha b B-\beta c l-N b) e_{2} \\
& +(\beta c N-\beta d M-a c-\alpha b C-\beta c m-N c) e_{3} \\
& +(-a d-\alpha b D-\beta c n-N d) e_{4}
\end{aligned}
$$




$$
\begin{aligned}
{[\mathcal{I}, \mathcal{I}]\left(e_{2}, e_{3}\right)=} & -\left[e_{2}, e_{3}\right]+\left[\sum A e_{i}, \sum k e_{i}\right]-\mathcal{I}\left[\sum A e_{i}, e_{3}\right]-\mathcal{I}\left[e_{2}, \sum k e_{i}\right] \\
= & A n e_{1}+B \alpha n e_{2}+C \beta n e_{3}-D k e_{1}-D \alpha e_{2}-D \beta m e_{3} \\
& +\mathcal{I}\left(D \beta e_{3}\right)-\mathcal{I}\left(n \alpha e_{2}\right) \\
= & A n e_{1}+B \alpha n e_{2}+C \beta n e_{3}-D k e_{1}-D \alpha l e_{2}-D \beta m e_{3} \\
& +\beta D\left(\sum k e_{i}\right)-\alpha n\left(\sum A e_{i}\right) \\
= & (A n-D k+\beta D k-\alpha n A) e_{1}+(\alpha B n-\alpha D l+\beta D l-\alpha B n) e_{2} \\
& +(\beta C n-\beta D m+\beta D m-\alpha n C) e_{3}+(\beta D n-\alpha D n) e_{4}
\end{aligned}
$$

$$
\begin{aligned}
{[\mathcal{I}, \mathcal{I}]\left(e_{2}, e_{4}\right)=} & -\left[e_{2}, e_{4}\right]+\left[\sum A e_{i}, \sum K e_{i}\right]-\mathcal{I}\left[\sum A e_{i}, e_{4}\right]-\mathcal{I}\left[e_{2}, \sum K e_{i}\right] \\
= & -\alpha e_{2}+A N e_{1}+B \alpha N e_{2}+C \beta N e_{3}-D K e_{1}-D \alpha L e_{2}-D \beta M e_{3} \\
& -\mathcal{I}\left(A e_{1}+\alpha B e_{2}+\beta C e_{3}\right)-\mathcal{I}\left(\alpha N e_{2}\right) \\
= & -\alpha e_{2}+A N e_{1}+B \alpha N e_{2}+C \beta N e_{3}-D K e_{1}-D \alpha L e_{2}-D \beta M e_{3} \\
& -A\left(\sum a e_{i}\right)-\alpha B\left(\sum A e_{i}\right)-\beta C\left(\sum k e_{i}\right)-\alpha N\left(\sum A e_{i}\right) \\
= & (A N-D K-A a-\alpha B A-\beta C k-\alpha N A) e_{1} \\
& +\left(-\alpha+\alpha B N-\alpha D L-A b-\alpha B^{2}-\beta C l-\alpha N B\right) e_{2} \\
& +(\beta C N-\beta D M-A c-\alpha B C-\beta C m-\alpha N C) e_{3} \\
& +(-A d-\alpha B D-\beta C n-\alpha N D) e_{4}
\end{aligned}
$$

$$
\begin{aligned}
{[\mathcal{I}, \mathcal{I}]\left(e_{3}, e_{4}\right)=} & -\left[e_{3}, e_{4}\right]+\left[\sum k e_{i}, \sum K e_{i}\right]-\mathcal{I}\left[\sum k e_{i}, e_{4}\right]-\mathcal{I}\left[e_{3}, \sum K e_{i}\right] \\
= & -\beta e_{3}+k N e_{1}+l \alpha N e_{2}+m \beta N e_{3}-n K e_{1}-n \alpha L e_{2}-n \beta M e_{3} \\
& -\mathcal{I}\left(k e_{1}+l \alpha e_{2}+m \beta e_{3}\right)-\mathcal{I}\left(N \beta e_{3}\right) \\
= & -\beta e_{3}+k N e_{1}+l \alpha N e_{2}+m \beta N e_{3}-n K e_{1}-n \alpha L e_{2}-n \beta M e_{3} \\
& -k\left(\sum a e_{i}\right)-\alpha l\left(\sum A e_{i}\right)-\beta m\left(\sum k e_{i}\right)-\beta N\left(\sum k e_{i}\right) \\
= & (k N-n K-k a-\alpha l A-\beta m k-\beta N k) e_{1} \\
& +(\alpha l N-\alpha n L-k b-\alpha l B-\beta m l-\beta N l) e_{2} \\
& +\left(-\beta+\beta m N-\beta n M-k c-\alpha l C-\beta m^{2}-\beta N m\right) e_{3} \\
& +(-k d-\alpha l D-\beta m n-\beta N n) e_{4}
\end{aligned}
$$

Since we are dealing with the case $-1<\alpha<\beta<1$ or $-1=\alpha<\beta<0$, the expressions for $[\mathcal{I}, \mathcal{I}]\left(e_{1}, e_{2}\right),[\mathcal{I}, \mathcal{I}]\left(e_{1}, e_{3}\right)$ and $[\mathcal{I}, \mathcal{I}]\left(e_{2}, e_{3}\right)$ give rise to the 
following system

$$
\left\{\begin{array}{l}
d A=0 \\
b D=0 \\
(\beta-1) D c+(\alpha-\beta) d C=0 \\
d D=0 \\
d k=0 \\
(\alpha-1) b n+(\beta-\alpha) d l=0 \\
c n=0 \\
d n=0 \\
(1-\alpha) A n+(\beta-1) D k=0 \\
D l=0 \\
C n=0 \\
D n=0
\end{array}\right.
$$

It is easy to show, as before, that this and inhomogeneous equations, coming from the computed expressions for $[\mathcal{I}, \mathcal{I}]\left(e_{1}, e_{4}\right),[\mathcal{I}, \mathcal{I}]\left(e_{2}, e_{4}\right)$ and $[\mathcal{I}, \mathcal{I}]\left(e_{3}, e_{4}\right)$, containing $-1,-\alpha,-\beta$, force $d=D=n=0$. The remaining torsion conditions result in the following system

$$
\left\{\begin{array}{l}
-1-a^{2}-\alpha b A-\beta c k=0 \\
(\alpha-1) b N-a b-\alpha b B-\beta c l=0 \\
(\beta-1) c N-a c-\alpha b C-\beta c m=0 \\
(1-\alpha) A N-A a-\alpha B A-\beta C k=0 \\
-\alpha-A b-\alpha B^{2}-\beta C l=0 \\
(\beta-\alpha) C N-A c-\alpha B C-\beta C m=0 \\
(1-\beta) k N-k a-\alpha l A-\beta m k=0 \\
(\alpha-\beta) l N-k b-\alpha l B-\beta m l=0 \\
-\beta-k c-\alpha l C-\beta m^{2}=0
\end{array}\right.
$$

Let us rewrite the homogeneous equations of (II) as

$$
\left\{\begin{array}{l}
b((\alpha-1) N-a-\alpha B)=\beta c l \\
A((1-\alpha) N-a-\alpha B)=\beta C k \\
c((\beta-1) N-a-\beta m)=\alpha b C \\
k((1-\beta) N-a-\beta m)=\alpha l A \\
C((\beta-\alpha) N-\alpha B-\beta m)=A c \\
l((\alpha-\beta) N-\alpha B-\beta m)=k b
\end{array} .\right.
$$


It is easy to prove that $A b c C k l \neq 0$. The reasoning always looks the same and uses (III) and inhomogeneous equations of (II). For example, if $A=0$ then from (III) we obtain $C k=0$, which is impossible in light of the first and the fifth equations of (II). Let us modify (III) a little bit into

(IV)

$$
\left\{\begin{array}{l}
A b((\alpha-1) N-a-\alpha B)=\beta A c l \\
A b((1-\alpha) N-a-\alpha B)=\beta b C k \\
c k((\beta-1) N-a-\beta m)=\alpha b C k \\
c k((1-\beta) N-a-\beta m)=\alpha A c l \\
C l((\beta-\alpha) N-\alpha B-\beta m)=A c l \\
C l((\alpha-\beta) N-\alpha B-\beta m)=b C k
\end{array}\right.
$$

Adding and subtracting each of the three pairs of two consecutive equations, after scalings, gives us the following equivalent system

$$
\left\{\begin{array}{l}
(-\alpha A b)(a+\alpha B)=\alpha \beta \frac{A c l+b C k}{2} \\
N=\frac{(\alpha \beta)(A c l-b C k)}{2(1-\alpha)(-\alpha A b)} \\
(-\beta c k)(a+\beta m)=\alpha \beta \frac{A c l+b C k}{2} \\
N=\frac{(\alpha \beta)(A c l-b C k)}{2(\beta-1)(-\beta c k)} \\
(-\alpha \beta C l)(\alpha B+\beta m)=\alpha \beta \frac{A c l+b C k}{2} \\
N=\frac{(\alpha \beta)(A c l-b C k)}{2(\alpha-\beta)(-\alpha \beta C l)}
\end{array}\right.
$$

Introducing new variables

$$
\begin{gathered}
x=(-\alpha A b), \\
y=(-\beta c k), \\
z=(-\alpha \beta C l), \\
\omega=\frac{(\alpha \beta)(A c l+b C k)}{2}, \\
\Omega=\frac{(\alpha \beta)(A c l-b C k)}{2}, \\
v=\alpha B, \\
w=\beta m,
\end{gathered}
$$


allows us to rewrite (V) as

$$
\left\{\begin{array}{l}
N=\frac{\Omega}{(1-\alpha) x} \\
N=\frac{\Omega}{(\beta-1) y} \\
N=\frac{\Omega}{(\alpha-\beta) z} \\
x(a+v)=\omega \\
y(a+w)=\omega \\
z(v+w)=\omega
\end{array},\right.
$$

and add the rewritten system coming from inhomogeneous equations

$$
\left\{\begin{array}{l}
1+a^{2}=x+y \\
\alpha^{2}+v^{2}=x+z \\
\beta^{2}+w^{2}=y+z
\end{array} .\right.
$$

Suppose that $\Omega=0$; then $\omega^{2}=-x y z$. Multiplying last two equations of (VI) we get

$$
x=-(a+w)(v+w),
$$

multiplying the fifth and the last equations of (VI) we get

$$
y=-(a+v)(v+w) \text {. }
$$

This gives

$$
x+y=-(v+w)(v+w+2 a) .
$$

Applying this in the first equation of (VII) leads to

$$
-(v+w)(v+w+2 a)=1+a^{2}
$$

which can be rewritten as $1=-(a+v+w)^{2}$, an obvious contradiction. We know then that $\Omega \neq 0$. From the first three equations of (VI) we get

$$
y=\frac{1-\alpha}{\beta-1} x \text { and } z=\frac{1-\alpha}{\alpha-\beta} x .
$$

Let us now apply this in the last three equations of (VI)

$$
\left\{\begin{array}{l}
x(a+v)=\omega \\
\frac{1-\alpha}{\beta-1} x(a+w)=\omega \\
\frac{1-\alpha}{\alpha-\beta} x(v+w)=\omega
\end{array},\right.
$$

and in system (VII) to obtain

$$
\text { (IX) }\left\{\begin{array}{l}
1+a^{2}=x+\frac{1-\alpha}{\beta-1} x=\frac{\beta-\alpha}{\beta-1} x \\
\alpha^{2}+v^{2}=x+\frac{1-\alpha}{\alpha-\beta} x=\frac{1-\beta}{\alpha-\beta} x \\
\beta^{2}+w^{2}=\frac{1-\alpha}{\beta-1} x+\frac{1-\alpha}{\alpha-\beta} x=\frac{(1-\alpha)(\alpha-\beta)+(1-\alpha)(\beta-1)}{(\beta-1)(\alpha-\beta)} x=\frac{-(1-\alpha)^{2}}{(\beta-1)(\alpha-\beta)} x
\end{array}\right.
$$


System (VIII) enables us to write

$$
a+v=\frac{1-\alpha}{\beta-1}(a+w) \text { and } a+v=\frac{1-\alpha}{\alpha-\beta}(v+w) .
$$

From the first equation

$$
v=\frac{1-\alpha}{\beta-1} a-a+\frac{1-\alpha}{\beta-1} w=\frac{2-\alpha-\beta}{\beta-1} a+\frac{1-\alpha}{\beta-1} w .
$$

Applying this in the second equation gives

$$
a+\frac{2-\alpha-\beta}{\beta-1} a+\frac{1-\alpha}{\beta-1} w=\frac{1-\alpha}{\alpha-\beta}\left(\frac{2-\alpha-\beta}{\beta-1} a+\frac{1-\alpha}{\beta-1} w\right)+\frac{1-\alpha}{\alpha-\beta} w
$$

which can be rewritten as

$$
\frac{(1-\alpha)(\alpha-\beta)-(1-\alpha)(2-\alpha-\beta)}{(\beta-1)(\alpha-\beta)} a=\frac{(1-\alpha)^{2}+(1-\alpha)(\beta-1)-(1-\alpha)(\alpha-\beta)}{(\beta-1)(\alpha-\beta)} w
$$

or simply as

$$
w=\frac{1-\alpha}{\alpha-\beta} a .
$$

Merging the first and the third equations of (IX) gives

$$
1+a^{2}=\frac{\beta-\alpha}{\beta-1} \frac{(\beta-1)(\alpha-\beta)}{-(1-\alpha)^{2}}\left(\beta^{2}+w^{2}\right)=\frac{(\alpha-\beta)^{2}}{(1-\alpha)^{2}}\left(\beta^{2}+\frac{(1-\alpha)^{2}}{(\alpha-\beta)^{2}} a^{2}\right)
$$

which results in

$$
1=\beta^{2} \frac{(\alpha-\beta)^{2}}{(1-\alpha)^{2}}
$$

There are two cases.

Suppose that $1-\alpha=\beta(\alpha-\beta)$. Then $\beta^{2}+(-\alpha) \beta+(1-\alpha)=0$, which implies that $\Delta=\alpha^{2}+4 \alpha-4 \geq 0$. Since $\alpha \in[-1,1)$, the last condition implies $\alpha>0$. Note also that $\Delta<\alpha^{2}$, which implies that $\beta$ cannot be equal to neither

$$
\frac{\sqrt{\Delta}+\alpha}{2}<\alpha
$$

since by an assumption $\beta>\alpha$, nor

$$
\frac{-\sqrt{\Delta}+\alpha}{2}<\alpha
$$

It must then be $(\alpha-1)=\beta(\alpha-\beta)$ or simply $\beta^{2}+(-\alpha)+(\alpha-1)=0$. In this case $\Delta=\alpha^{2}-4 \alpha+4=(2-\alpha)^{2}$ and, again, $\beta$ cannot be equal to neither

$$
\frac{2-\alpha+\alpha}{2}=1
$$

nor

$$
\frac{\alpha-2+\alpha}{2}=\alpha-1<\alpha .
$$


This final contradiction shows that our original assumption about the existence of a zero torsion endomorphism must be false and the proof is completed.

REMARK 4.5. There is no complex structure on the Lie algebras $A_{4,2}^{\alpha} \times \mathfrak{h}$ for $\alpha \neq 1, A_{4,3} \times \mathfrak{h}, A_{4,4} \times \mathfrak{h}$ or $A_{4,5}^{\alpha, \beta} \times \mathfrak{h}$ for $-1<\alpha<\beta<1$ or $-1=\alpha<\beta<0$ and any Lie algebra $\mathfrak{h}$.

The next theorem deals with the only nilpotent Lie algebra in this section: $A_{4,1} \times \mathbb{R}^{2}$. Non-existence of a complex structure on it was proven by Salamon in [19]; here we give a different proof. In parallel we deal with the algebra $A_{4,1} \times A_{2,2}$, which has to be analyzed another way since it is not nilpotent.

Proposition 4.6. There is no complex structure on the Lie algebras $A_{4,1} \times$ $\mathbb{R}^{2}$ and $A_{4,1} \times A_{2,2}$, although there is a zero torsion endomorphism on $A_{4,1}$.

Proof. The proof goes simultaneously for both cases. Assume that $\mathcal{J}$ is a complex structure on one of the given algebras. In both cases

$$
\mathcal{J}\left(A_{4,1}\right) \cap A_{4,1}
$$

is at least two dimensional since it is $\mathcal{J}$ invariant and these spaces cannot be disjoint given their dimensions. This in turn means that

$$
\mathcal{I}:=\mathcal{J}_{1}^{1}
$$

defined as in Lemma 2.1, has eigenvalues $i$ and $-i$. Let us write again the torsion conditions in order to get more information on the endomorphism $\mathcal{I}$.

$$
\begin{aligned}
{[\mathcal{I}, \mathcal{I}]\left(e_{1}, e_{2}\right)=} & -\left[e_{1}, e_{2}\right]+\left[\sum a e_{i}, \sum A e_{i}\right]-\mathcal{I}\left[\sum a e_{i}, e_{2}\right]-\mathcal{I}\left[e_{1}, \sum A e_{i}\right] \\
= & b D e_{1}+c D e_{2}-d B e_{1}-d C e_{2}+\mathcal{I}\left(d e_{1}\right) \\
= & b D e_{1}+c D e_{2}-d B e_{1}-d C e_{2}+d\left(\sum a e_{i}\right) \\
= & (b D-d B+d a) e_{1}+(c D-d C+d b) e_{2}+(d c) e_{3}+\left(d^{2}\right) e_{4} \\
{[\mathcal{I}, \mathcal{I}]\left(e_{1}, e_{3}\right)=} & -\left[e_{1}, e_{3}\right]+\left[\sum a e_{i}, \sum k e_{i}\right]-\mathcal{I}\left[\sum a e_{i}, e_{3}\right]-\mathcal{I}\left[e_{1}, \sum k e_{i}\right] \\
= & b n e_{1}+c n e_{2}-d l e_{1}-d m e_{2}+\mathcal{I}\left(d e_{2}\right)=b n e_{1}+c n e_{2}-d l e_{1} \\
& -d m e_{2}+d\left(\sum A e_{i}\right) \\
= & (b n-d l+d A) e_{1}+(c n-d m+d B) e_{2}+(d C) e_{3}+(d D) e_{4} \\
{[\mathcal{I}, \mathcal{I}]\left(e_{1}, e_{4}\right)=} & -\left[e_{1}, e_{4}\right]+\left[\sum a e_{i}, \sum K e_{i}\right]-\mathcal{I}\left[\sum a e_{i}, e_{4}\right]-\mathcal{I}\left[e_{1}, \sum K e_{i}\right] \\
= & b N e_{1}+c N e_{2}-d L e_{1}-d M e_{2}-\mathcal{I}\left(b e_{1}+c e_{2}\right) \\
= & b N e_{1}+c N e_{2}-d L e_{1}-d M e_{2}-b\left(\sum a e_{i}\right)-c\left(\sum A e_{i}\right) \\
= & (b N-d L-b a-c A) e_{1}+\left(c N-d M-b^{2}-c B\right) e_{2} \\
& +(-b c-c C) e_{3}+(-b d-c D) e_{4}
\end{aligned}
$$




$$
\begin{aligned}
{[\mathcal{I}, \mathcal{I}]\left(e_{2}, e_{3}\right)=} & -\left[e_{2}, e_{3}\right]+\left[\sum A e_{i}, \sum k e_{i}\right]-\mathcal{I}\left[\sum A e_{i}, e_{3}\right]-\mathcal{I}\left[e_{2}, \sum k e_{i}\right] \\
= & B n e_{1}+C n e_{2}-D l e_{1}-D m e_{2}+\mathcal{I}\left(D e_{2}\right)-\mathcal{I}\left(n e_{1}\right) \\
= & B n e_{1}+C n e_{2}-D l e_{1}-D m e_{2}+D\left(\sum A e_{i}\right)-n\left(\sum a e_{i}\right) \\
& =(B n-D l+D A-n a) e_{1}+(C n-D m+D B-n b) e_{2} \\
& +(D C-n c) e_{3}+\left(D^{2}-n d\right) e_{4} \\
{[\mathcal{I}, \mathcal{I}]\left(e_{2}, e_{4}\right)=} & {\left[e_{2}, e_{4}\right]+\left[\sum A e_{i}, \sum K e_{i}\right]-\mathcal{I}\left[\sum A e_{i}, e_{4}\right]-\mathcal{I}\left[e_{2}, \sum K e_{i}\right] } \\
= & -e_{1}+B N e_{1}+C N e_{2}-D L e_{1}-D M e_{2}-\mathcal{I}\left(B e_{1}+C e_{2}\right)-\mathcal{I}\left(N e_{1}\right) \\
= & -e_{1}+B N e_{1}+C N e_{2}-D L e_{1}-D M e_{2}-B\left(\sum a e_{i}\right) \\
& -C\left(\sum A e_{i}\right)-N\left(\sum a e_{i}\right) \\
= & (-1+B N-D L-B a-C A-N a) e_{1} \\
& +(C N-D M-B b-C B-N b) e_{2} \\
& +\left(-B c-C{ }^{2}-N c\right) e_{3}+(-B d-C D-N d) e_{4} \\
= & -\left[e_{3}, e_{4}\right]+\left[\sum k e_{i}, \sum K e_{i}\right]-\mathcal{I}\left[\sum k e_{i}, e_{4}\right]-\mathcal{I}\left[e_{3}, \sum K e_{i}\right] \\
= & -e_{2}+l N e_{1}+m N e_{2}-n L e_{1}-m N e_{2}-\mathcal{I}\left(l e_{1}+m e_{2}\right)-\mathcal{I}\left(N e_{2}\right) \\
= & -e_{2}+l N e_{1}+m N e_{2}-n L e_{1}-n M e_{2}-l\left(\sum a e_{i}\right)-m\left(\sum A e_{i}\right) \\
& -N\left(\sum A e_{i}\right) \\
= & (l N-n L-l a-m A-N A) e_{1} \\
& +(-1+m N-n M-l b-m B-N B) e_{2} \\
& +(-l c-m C-N C) e_{3}+(-l d-m D-N D) e_{4}
\end{aligned}
$$

Combining all obtained equations we end with the system

$$
\left\{\begin{array}{l}
D=d=c n=b n=C n=0 \\
c N-b^{2}-c B=0 \\
b N-b a-c A=0 \\
b c+c C=0 \\
B n=n a \\
-1+B N-B a-C A-N a=0 \\
C N-B b-C B-N b=0 \\
-B c-C^{2}-N c=0 \\
l N-n L-l a-m A-N A=0 \\
-1+m N-n M-l b-m B-N B=0 \\
-l c-m C-N C=0
\end{array}\right.
$$


Suppose that $n \neq 0$. Then $C=0$ and $B=a$, which implies that the sixth equation states $-1-a^{2}=0$. Suppose that $c=0$; then $b=C=0$ and this means that the matrix of $\mathcal{I}$ is

$$
\left[\begin{array}{cccc}
a & A & k & K \\
0 & B & l & L \\
0 & 0 & m & M \\
0 & 0 & 0 & N
\end{array}\right]
$$

which has only real eigenvalues. This contradicts our remark from the beginning of the proof. For now we have established that $d=D=n=0$ and $c \neq 0$. From the fourth equation it follows that $b=-C$, subtracting the second equation from the eighth one gives $N=0$. We now see that

$$
\mathcal{J}\left(A_{4,1}\right) \cap A_{4,1} \subset \operatorname{span}\left\{e_{1}, e_{2}, e_{3}\right\} .
$$

From this and the remark from the beginning of the proof we conclude that there are two linearly independent vectors

$$
u, v \in \operatorname{span}\left\{e_{1}, e_{2}, e_{3}\right\}
$$

such that

$$
\mathcal{J} u=v
$$

From the torsion condition we obtain

$$
0=[\mathcal{J}, \mathcal{J}]\left(u, e_{4}\right)=-\left[u, e_{4}\right]-\mathcal{J}\left[v, e_{4}\right]
$$

or just

$$
\mathcal{J}\left[v, e_{4}\right]=-\left[u, e_{4}\right] .
$$

Since $u, v$ are linearly independent, at most one of them can belong to

$$
\operatorname{span}\left\{e_{1}\right\} \text {. }
$$

From the condition

$$
\mathcal{J}\left[v, e_{4}\right]=-\left[u, e_{4}\right]
$$

we see that actually none of them does. From all of these we obtain

$$
\left[v, e_{4}\right] \text { and }-\left[u, e_{4}\right]
$$

form a basis of

$$
\operatorname{span}\left\{e_{1}, e_{2}\right\}
$$

which turns out to be an $\mathcal{J}$ invariant subspace, contradicting the fact that $c \neq$ 0 . For a zero torsion endomorphism on $A_{4,1}$ just take $a=-1$ and $m=N=1$ while the other variables are zero.

We are ready to summarize the results obtained in this section. 
THEOREM 4.7. Using the previous convention, the classification of the Lie algebras of the form $\mathfrak{g} \times \mathbb{R}^{2}$ and $\mathfrak{g} \times A_{2,2}$, for an indecomposable four dimensional Lie algebra $\mathfrak{g}$, admitting complex structures is presented in the table below.

\begin{tabular}{|c|c|c|}
\hline $\mathfrak{g} \times \mathfrak{h}$ & $\mathbb{R}^{2}$ & $A_{2,2}$ \\
\hline$A_{4,1}$ & $x$ & $x$ \\
\hline$A_{4,2}^{1}$ & $\checkmark$ & $\checkmark$ \\
\hline$A_{4,2}^{\alpha}, \alpha \neq 0,1$ & $x$ & $x$ \\
\hline$A_{4,3}$ & $x$ & $x$ \\
\hline$A_{4,4}$ & $x$ & $x$ \\
\hline$A_{4,5}^{\alpha, \beta}, \alpha \beta \neq 0$ and $-1<\alpha<\beta<1$ or $-1=\alpha<\beta<0$ & $x$ & $x$ \\
\hline$A_{4,5}^{\alpha, \alpha}, \alpha \neq 0,-1 \leq \alpha \leq 1$ & $\checkmark$ & $\checkmark$ \\
\hline$A_{4,5}^{\alpha, 1}, \alpha \neq 0,-1<\alpha<1$ & $\checkmark$ & $\checkmark$ \\
\hline$A_{4,6}^{\alpha, \beta}, \alpha \neq 0, \beta \geq 0$ & $\checkmark$ & $\checkmark$ \\
\hline$A_{4,7}$ & $\checkmark$ & $\checkmark$ \\
\hline$A_{4,8}$ & $\checkmark$ & $\checkmark$ \\
\hline$A_{4,9}^{\beta},-1<\beta \leq 1$ & $\checkmark$ & $\checkmark$ \\
\hline$A_{4,10}$ & $\checkmark$ & $\checkmark$ \\
\hline$A_{4,11}^{\alpha}, \alpha>0$ & $\checkmark$ & $\checkmark$ \\
\hline$A_{4,12}$ & $\begin{array}{l}\checkmark \\
\checkmark\end{array}$ & $\begin{array}{l} \\
\checkmark\end{array}$ \\
\hline
\end{tabular}

5. The case of $\mathfrak{g}_{5} \times \mathbb{R}$. We intend to give here a short discussion on the last possible form of a decomposable six-dimensional Lie algebra, namely $\mathfrak{g} \times \mathbb{R}$ for a five dimensional indecomposable Lie algebra $\mathfrak{g}$. In this case examining the existence of zero torsion endomorphisms on $\mathfrak{g}$ is much more complicated and there is much bigger variety of algebras to be studied, cf. [18]. We propose another attempt towards the classification problem in this class. The author knows no example of a five dimensional indecomposable Lie algebra $\mathfrak{g}$ for which $\mathfrak{g} \times \mathbb{R}$ would admit a complex structure but not admit the canonical complex structure associated with some normal almost contact structure on $\mathfrak{g}$. This leads us to the following question.

Question 5.1. For a five dimensional indecomposable Lie algebra $\mathfrak{g}$, does $\mathfrak{g} \times \mathbb{R}$ admit a complex structure if and only if $\mathfrak{g}$ admit a normal almost contact structure?

Note that due to the results presented in Sections 2 and 3, the answer is affirmative for $\mathfrak{g}$ being three dimensional and we suspect that the same also holds in the above situation. If true, this would in turn allow us to confine discussion to the existence of special kind zero torsion endomorphisms on $\mathfrak{g}$. 


\section{References}

1. Andrada A., Barberis M.L., Dotti I., Classification of abelian complex structures on 6-dimensional Lie algebras, J. Lond. Math. Soc. (2), 83 (2011), 232-255.

2. Andrada A., Barberis M.L., Dotti I., Corrigendum: Classification of abelian complex structures on 6-dimensional Lie algebras, J. Lond. Math. Soc. (2), 87 (2013), 319-320.

3. Angella D., Otal A., Ugarte L., Villacampa R., Complex structures of splitting type, Rev. Mat. Iberoam., 33(4) (2017), 1309-1350.

4. Bianchi L., Sugli spazi a tre dimensioni che ammettono un gruppo continuo di movimenti, Mem. Soc. Ital. Scienze (3), 11 (1897), 267-352.

5. Blair D.E., Riemannian Geometry of Contact and Symplectic Manifolds, Progress in Math. 203, Birkhäuser, Boston, 2002.

6. Bock Ch., On low-dimensional solvmanifolds, Asian J. Math., 20(2) (2016), 199-262.

7. Ceballos M., Otal A., Ugarte L., Villacampa R., Invariant complex structures on 6-nilmanifolds: classification, Frölicher spectral sequence and special Hermitian metrics, J. Geom. Anal., 26 (2016), 252-286.

8. Chevalley C., Eilenberg S., Cohomology theory of Lie groups and Lie algebras, Trans. Amer. Math. Soc. 63 (1948), 85-124.

9. Czarnecki A., Sroka M., Six-dimensional product Lie algebras admitting integrable complex structures, J. Pure Appl. Algebra, 222 (2018), 1111-1125.

10. Fino A., Otal A., Ugarte L., Six-dimensional solvmanifolds with holomorphically trivial canonical bundle, Int. Math. Res. Not. IMRN, 24 (2015), 13757-13799.

11. Freibert M., Schulte-Hengesbach F., Half-flat structures on decomposable Lie groups, Transform. Groups, 17(1) (2012), 123-141.

12. Magnin L., Left invariant complex structures on $U(2)$ and $S U(2) \times S U(2)$ revisited, Rev. Roumaine Math. Pures Appl., 55 (2010), 269-296.

13. Morimoto A., Structures complexes sur les groupes de Lie semi-simples, C. R. Acad. Sci. Paris, 242 (1956), 1101-1103.

14. Morimoto A., On normal almost contact structures, J. Math. Soc. Japan, 15 (1963), 420-436.

15. Mubarakzjanov G.M., Classification of real structures of Lie algebras of order fifth (in Russian), Izv. Vyss. Ucebn. Zaved. Matematika, 35 (1963), 104-116.

16. Nomizu K., On the cohomology of compact homogeneous spaces of nilpotent Lie groups, Ann. of Math. (2), 59 (1954), 531-538.

17. Ovando G., Invariant complex structures on solvable real Lie groups, Manuscripta Math., 103 (2000), 19-30.

18. Patera J., Sharp R.T., Winternitz P., Zassenhaus H., Invariants of real low dimension Lie algebras, J. Mathematical Phys., 17 (1976), 986-994.

19. Salamon S.M., Complex structures on nilpotent Lie algebras, J. Pure Appl. Algebra, 157 (2001), 311-333.

20. Samelson H., A class of complex analytic manifolds, Portugal. Math., 12 (1953), 129-132.

21. Schulte-Hengesbach F., Half-flat structures on products of three-dimensional Lie groups, J. Geom. Phys., 60(11) (2010), 1726-1740.

22. Snow D.M., Invariant complex structures on reductive Lie groups, J. Reine Angew. Math., 371 (1986), 191-215.

23. Snow J.E., Invariant complex structures on four-dimensional solvable real lie groups, Manuscripta Math., 66 (1990), 397-412.

24. Wang H.C., Closed manifolds with homogeneous complex structure, Amer. J. Math., 76 (1954), 1-32. 
Received November 9, 2020

\author{
Marcin Sroka \\ Faculty of Mathematics and Computer Science \\ Jagiellonian University \\ Łojasiewicza 6 \\ 30-348 Kraków \\ Poland \\ e-mail: Marcin.Sroka@im.uj.edu.pl
}

